\title{
Review
}

\section{Can Human Pluripotent Stem Cell-Derived Cardiomyocytes Advance Understanding of Muscular Dystrophies?}

\author{
Spandan Kalra ${ }^{\mathrm{a}}$, Federica Montanaro ${ }^{\mathrm{b}, *}$ and Chris Denning ${ }^{\mathrm{a}, *}$ \\ ${ }^{a}$ Department of Stem Cell Biology, Centre for Biomolecular Sciences, University of Nottingham, UK \\ ${ }^{\mathrm{b}}$ Dubowitz Neuromuscular Centre, Department of Molecular Neurosciences, \\ University College London - Institute of Child Health, London, UK
}

\begin{abstract}
Muscular dystrophies (MDs) are clinically and molecularly a highly heterogeneous group of single-gene disorders that primarily affect striated muscles. Cardiac disease is present in several MDs where it is an important contributor to morbidity and mortality. Careful monitoring of cardiac issues is necessary but current management of cardiac involvement does not effectively protect from disease progression and cardiac failure. There is a critical need to gain new knowledge on the diverse molecular underpinnings of cardiac disease in MDs in order to guide cardiac treatment development and assist in reaching a clearer consensus on cardiac disease management in the clinic. Animal models are available for the majority of MDs and have been invaluable tools in probing disease mechanisms and in pre-clinical screens. However, there are recognized genetic, physiological, and structural differences between human and animal hearts that impact disease progression, manifestation, and response to pharmacological interventions. Therefore, there is a need to develop parallel human systems to model cardiac disease in MDs. This review discusses the current status of cardiomyocytes (CMs) derived from human induced pluripotent stem cells (hiPSC) to model cardiac disease, with a focus on Duchenne muscular dystrophy (DMD) and myotonic dystrophy (DM1). We seek to provide a balanced view of opportunities and limitations offered by this system in elucidating disease mechanisms pertinent to human cardiac physiology and as a platform for treatment development or refinement.
\end{abstract}

Keywords: Human embryonic stem cells, human induced pluripotent stem cells, Cas9/CRISPR genome editing, cardiomyocytes, Duchenne muscular dystrophy (DMD), myotonic dystrophy (DM1), disease modelling, exon skipping, gene therapy

\section{INTRODUCTION}

The muscular dystrophies (MDs) are clinically and molecularly a highly heterogeneous group of single-gene disorders that are characterised clinically

\footnotetext{
*Correspondence to: Chris Denning, Department of Stem Cell Biology, Centre for Biomolecular Sciences, University of Nottingham, NG7 2RD, UK. E-mail: chris.denning@ nottingham.ac.uk and Federica Montanaro, Dubowitz Neuromuscular Centre, Department of Molecular Neurosciences, University College London - Institute of Child Health, London, WC1N 1EH, UK. E-mail: f.montanaro@ucl.ac.uk.
}

by progressive skeletal muscle weakness and wasting [1]. Transmission is usually by inheritance but de novo mutations are common, making it impossible to fully eradicate the disease. The study of disease mechanisms and treatment development has primarily focused on skeletal muscles with the goal of preserving patient mobility and preventing respiratory failure. However, cardiac disease is present in several MDs (Table 1) and has emerged as an increasingly important contributor to morbidity and mortality [2, 3]. Current guidelines for management of cardiac disease in MDs highlight the importance 


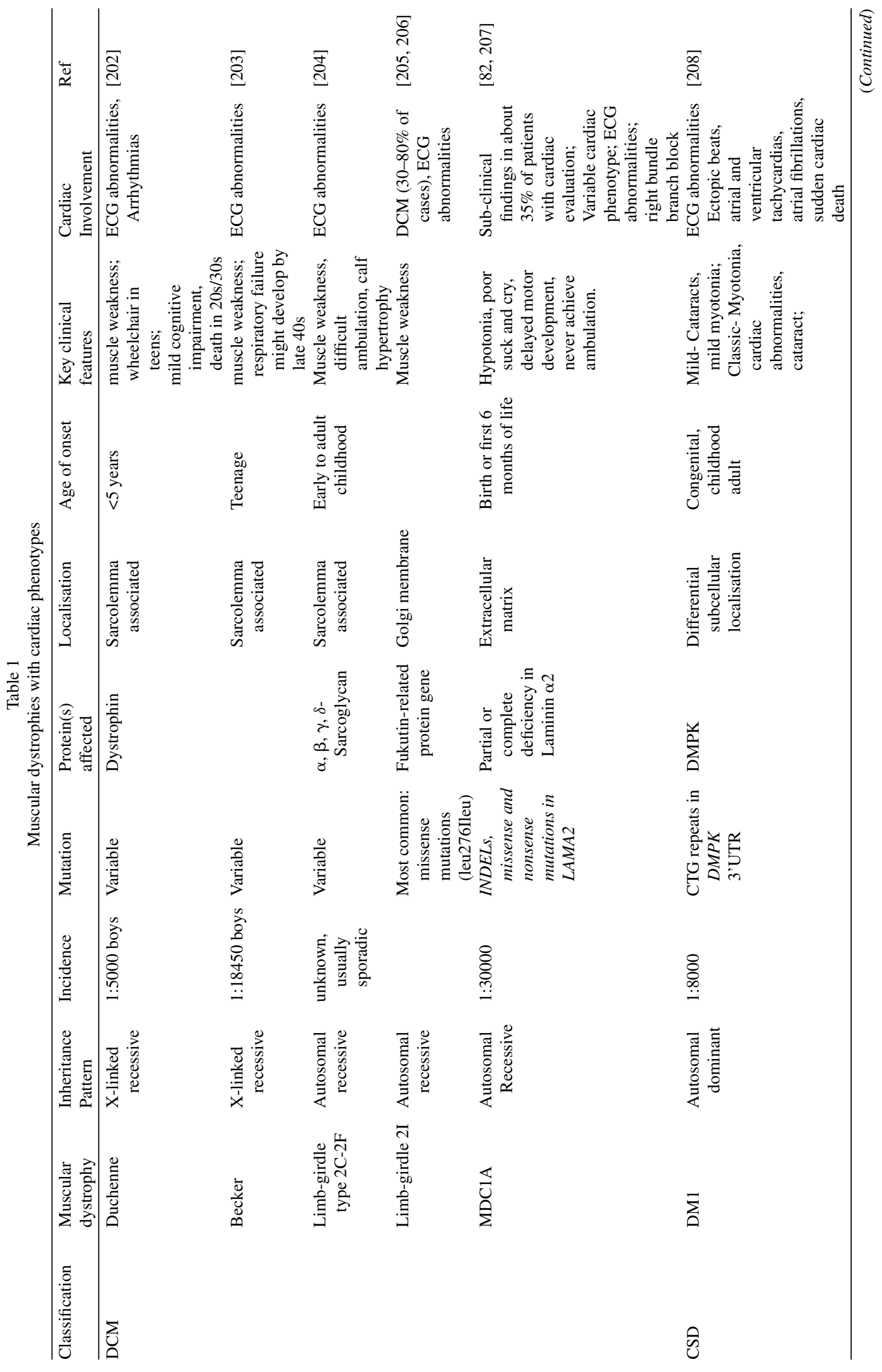




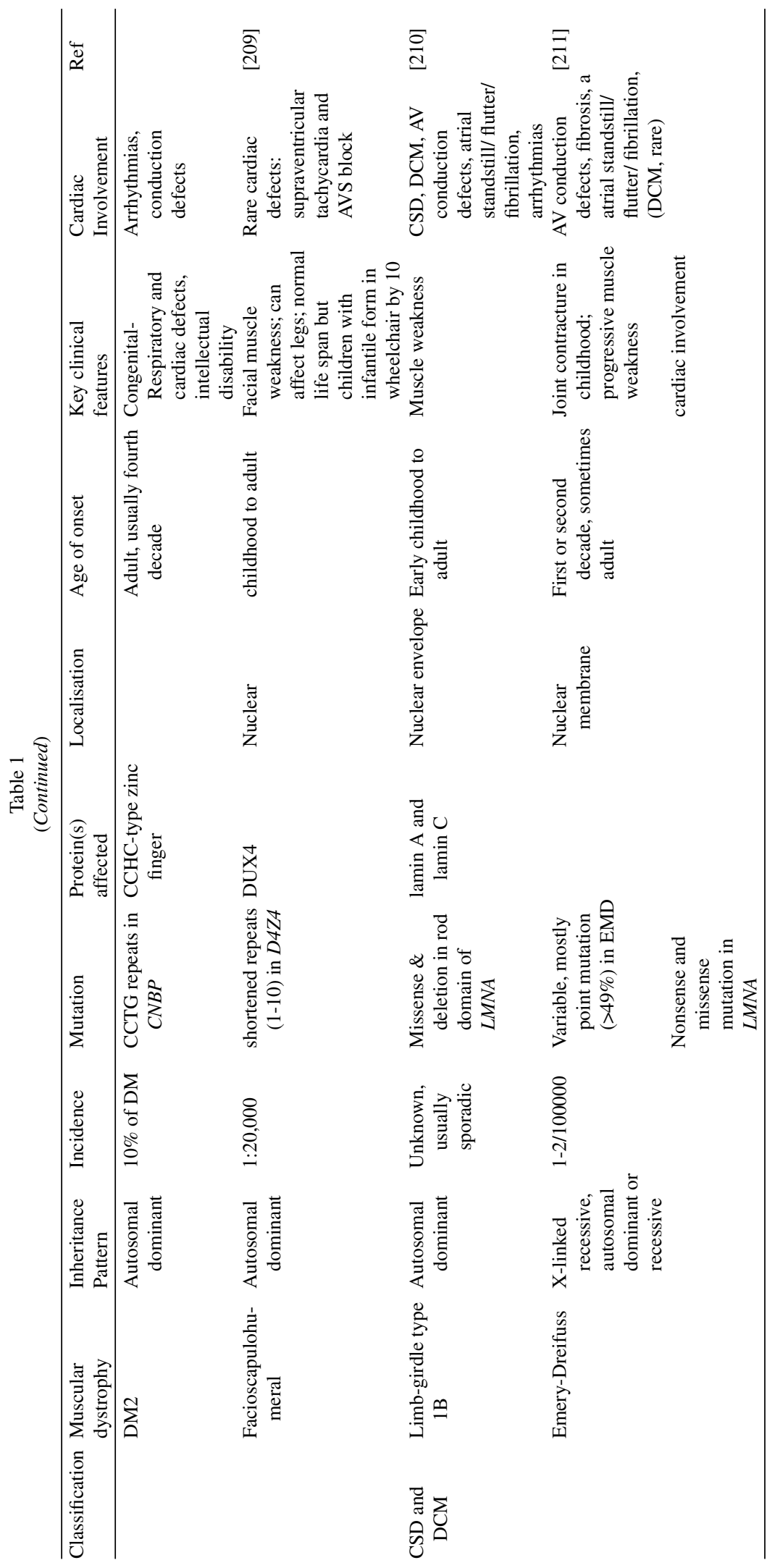


of careful monitoring and tend to favour early intervention with cardio-protective drugs or pacemakers, sometimes even before patients become symptomatic [4]. However, a clear consensus on management of cardiac involvement and timing of treatment has not been recognised. Furthermore, current interventions are insufficient to prevent cardiac failure and are not effective in all patients. Molecular processes underlying cardiac disease in MDs are not fully understood, and a current challenge is to determine whether promising therapeutics can translate from skeletal to cardiac muscle. The biology of these muscles is very different regarding physical, mechanical and signalling mechanisms. Notably, there is often no correlation between the severity, or even presence, of disease between cardiac and skeletal muscles [4-7], suggesting different underlying disease mechanisms exist between tissue types.

Several reviews summarize [8] our current understanding of disease mechanisms and responses to various treatments that have been derived from animal models of MDs [9-14]. However, in the case of cardiac physiology and pharmacology, there are known differences between humans and animals. Therefore, there is a critical need to develop humanbased platforms to study cardiac disease mechanisms and treatment responses relevant to the human heart. In this review, we specifically focus on the current status and future potential of human induced pluripotent stem cells (hiPSCs) and their differentiation into cardiomyocytes (CMs) to further our understanding of human cardiac disease mechanisms in MDs, and as a platform for new treatment development. Among all MDs with cardiac involvement, hiPSC derived CMs (hiPSC-CMs) have been generated for Duchenne muscular dystrophy (DMD) and myotonic dystrophy type 1 (DM1). DMD and DM1 are also excellent examples of the two different major manifestations of cardiac involvement in MDs: Dilated cardiomyopathy (DCM) and conduction system disease (CSD), respectively. These two modalities of cardiac disease present different challenges and opportunities relative to hiPSC modelling, which will be discussed in detail later.

\section{Why develop hiPSC-based model system?}

\section{Differences in cardiac physiology between animals and humans}

Animal models have been generated for most MDs and have been instrumental in our understanding of cardiac disease in MDs. However, there are instances where animal models do not faithfully replicate the severity of cardiac disease seen in patients. For example, the $m d x$ mouse, the most commonly used animal model for DMD, develops mild cardiac disease only late in life and has a near normal lifespan [13]. By contrast, cardiac involvement begins very early in DMD patients (17\% of patients are already affected before 10 years of age [15]) and is a major contributor to early death. In other instances, animal models are not genetic equivalents to the human condition because introduction of the human mutation alone does not cause disease or is genetically unstable. For example, modelling DM1 cardiac disease in lower organisms (C. elegans, Drosophila) or mice requires supra-physiological expression levels or longer triple repeat tracts to cause disease $[16,17]$. Furthermore, the repeat expansion is unstable in mice and undergoes a reduction instead of further expansion from one generation to the next, which is the opposite to the human condition [17].

Additionally, differences in the structure and physiology of the heart between species have an important bearing on how this organ responds to genetic mutations and to applied treatments. This is particularly highlighted in the mouse [18], the animal model most readily available to study MDs. The beat rate of the mouse heart is $\sim 10$ times faster than in humans (500bpm vs 60bpm) and the electrocardiogram duration is 5-10 times shorter $(50-100 \mathrm{~ms}$ in the mouse versus $450 \mathrm{~ms}$ in humans). While an increase in heart rate is associated with increased force of contraction in humans, it is associated with decreased force in mice [19]. The ion channels that regulate repolarisation of the CM plasma membrane are also different between mice and humans. In mice, $\mathrm{CM}$ repolarisation is driven primarily by $\mathrm{I}_{\mathrm{to}}, \mathrm{I}_{\mathrm{K} \text {, slow1 }}, \mathrm{I}_{\mathrm{K} \text {,slow2 }}$, $\mathrm{I}_{\mathrm{SS}}$ ion channels, while in humans this role is achieved by the potassium channels, $\mathrm{I}_{\mathrm{Kr}}$ and $\mathrm{I}_{\mathrm{Kr}}$ [20]. Differences also exist in the expression of structural genes both during development and in the mature heart. Developmental myosin light chains, MLC2a and MLC2 $\mathrm{v}$ are differentially expressed and localized during development between rodents and humans [21]. In the mature human heart, expression of alpha and beta myosin heavy chains $(\alpha-/ \beta-M H C)$ locates to the atria and ventricles, respectively, but in the mouse $\alpha \mathrm{MHC}$ is expressed in both locations $[21,22]$. Phospholamban, a key regulator of intracellular calcium concentration during contraction plays different roles in mice and humans [21, 23]. A greater complexity of alternative splicing has been reported in humans relative to rodents [24], and appears to 
underlie some of the observed differences in pharmacological properties [25], cardiac contractility and disease progression $[26,27]$. These inter-species differences have important implications not only for cardiac mechanics and response to disease, but also for drug responses and toxicology. For example, mice are at least ten times more tolerant to $37 \%$ of drugs than humans. Issues extend to rats and dogs, that tolerate 4.5 - to 100 -fold the concentration of various chemotherapeutic agents than humans (e.g. ThioTEPA, Myleran, Actinomycin-D, Mitomycin C, Mithramycin, Fludarabine) [28]. Therefore, animal models are an imperfect correlate of human cardiac disease and, most important for development of new treatments, they are often poor predictors of drug efficacy, dosing and toxicology in humans.

\section{Advantages of hiPSCs relative to other sources of human CMs}

Ideally, the existing animal model systems would be complemented by a source of human CMs harbouring patient-relevant mutations. This has been achieved for skeletal muscle by taking muscle biopsies. Cardiac biopsies are not taken from MD patients because removing heart tissue is high risk and lacks direct benefit to the patient since diagnosis can be achieved by less invasive routes (e.g. skeletal muscle biopsy or blood sample). Studies on cardiac biopsies or isolated primary human CMs primarily rely on tissue obtained from transplants that, by virtue, is from the end-stage failing heart and may not be representative of the early pathological changes that occur in CMs. Alternative indirect sources of human CMs include trans-differentiation of human skin fibroblasts using combinations of the transcription factors GATA4, TBX5 and MEF2C. However, the efficiency $(1-25 \%)$ is too low to be useful [29-31]. Thus, currently, the most realistic way to generate human CMs from a broad range of genotypes relevant to the muscular dystrophies is via differentiation from hiPSCs.

hiPSCs can be generated from various sources using minimally-invasive procedures. Examples include fibroblasts isolated from skin biopsies or stem cells isolated from urine (most likely of glomerular parietal epithelial origin) [32-34]. As a result, both patients and healthy controls tend to be willing to donate cells for banking to be used for diagnostic and research purposes. The initial inefficiencies in hiPSC reprogramming and hiPSC-CM differentiation methods that hindered progress have now been overcome by stepwise improvements in the technologies available. For example, non-integrating methods approaches have replaced integrating retrovirus [34] to improve hiPSC reprogramming efficiencies by 44 fold from $0.1 \%$ to $\sim 4.4 \%$ [35].

Improvements in the methods that underpin hiPSC technologies are allowing considerable expansion of the range of patient mutations that can be modelled. It is now conceivable that a reasonable proportion of the $>7000$ documented mutations reported for the $D M D$ gene [36] could be modelled in hiPSC, something that is not realistic in animal models. Indeed, large scale banking schemes including Human Induced Pluripotent Stem Cells Initiative (HIPSCI), StemBANCC/IMI, California Institute for Regenerative Medicine and New York Stem Cell Foundation will create hiPSC lines from 7,000 normal or diseased skin biopsy donors using Sendaivirus, episomes or mRNA with a combination of SOX2, c-MYC, OCT4, KLF4 and/or LIN28 [37].

In parallel, improvements in cardiac differentiation protocols have increased efficiency from $1 \%$ to $98 \%$ (reviewed previously in [21]). This has been achieved by identifying signalling pathways that sequentially regulate key steps in cardiac development in vivo, including pathways involving transforming growth factor family members (TGF- $\beta$; bone morphogenetic proteins [BMP] and Activin A), fibroblast growth factors (bFGF; FGF2) and Wingless (WNT). Translating these developmental signals to hiPSCs in vitro has been empirical and iterative but has defined the concentrations and timings of growth factors and/or small molecules that activate or inhibit relevant cardiogenic pathways. Thus, the relative ease of efficient reprogramming and directed cardiogenesis is accelerating progress towards biomedical application.

\section{hiPSCs capture genome diversity}

The ability to efficiently produce hiPSC-CMs represents an essential development to model human cardiac disease. This technology is now being coupled with advances in precise genome engineering. This allows the introduction or correction of almost any mutation of interest [38] and overcomes the issues of cost, time and species differences associated with the generation and study of animal models for the myriad of patient mutations. In addition, it enables the study of mutations in the unique genetic environment of the individual patient, allowing for genome-mutation interactions to be revealed.

Initially, genetic modification of human pluripotent stem cells (hPSCs) was challenging but the improvements in culture systems allowed transfection and viral transduction efficiencies of $>50 \%$ and 
95\%, respectively [39]. Additionally, low recombination frequencies ( 1 in $10^{6}-10^{9}$ cells) in most mammalian cells prevented widespread take-up of gene targeting. With the advent of nuclease-mediated approaches including zinc finger nucleases [40, 41] and transcription activator-like effector nucleases $[42,43]$ improved the targeting efficiencies by up to 2000- fold. However, the greatest breakthrough in genome engineering came with the development of the Cas9/CRISPR (Clustered Regularly Interspaced Short Palindromic Repeat) system [44]. This allowed correction of individual patient mutations to create isogenic controls in which only the mutated sequences differ in an otherwise unchanged genetic background. Conversely, it is also possible to introduce different genetic mutations in a control hiPSC lines, allowing the study of mutation-specific effects in a controlled genetic environment. Kim et al. [45] showed up to $79 \%$ targeting efficiency in CCR5 locus in human cells, including human embryonic stem cells (hESCs). The efficiency of the approach is at the level that projects are underway to knock out every gene in the mouse genome; it is likely that similar projects will follow in hPSCs. Therefore, hiPSCs are ideally positioned for personalised medicine, but can in theory also facilitate the study and identification of disease modifiers [2].

\section{hiPSCs for drug development and toxicology studies}

In the 13 years from 2000-2013, pharmacological responses of hiPSC-CMs to only 60 different compounds had been demonstrated [18] but single reports now evaluate over 130 compounds [46]. Assessment by AstraZeneca [47], J\&J [48] and GlaxoSmithKline [49] have shown that hiPSC-CMs can detect cardiotoxicity at an accuracy of 70-90\% and rival predictivity of primary cells isolated from $\mathrm{dog}$ and rabbit hearts [50]. Notably, hiPSC-CMs were used to show that toxicity was reduced when the anticancer drug, doxorubicin, was delivered via a HER2-targeted liposomal pathway; this assisted the decision to advance to Phase I testing [51]. Such studies have led the CIPA initiative (Comprehensive In Vitro Proarrhythmia Assay) to propose integration of hiPSC-CMs into the ICH (International Conference on Harmonisation) S7a/b and E14 guidelines by the end of 2015 . These guidelines have been the mainstay over the last decade of preclinical assessment of cardiac electrophysiology for new drugs [52]. These observations have continued into phenotypic and pharmacological assessment of hiPSC-CM models of various conditions [18]. This includes the disorders that affect electrophysiology, structure, contractility and survival (see below and refs: [53-64]. Most notably, the inability to manage effectively treatment of an individual with complex LQTS was addressed by deriving hiPSC-CMs and performing multi-parameter in vitro drug testing to better define the underlying molecular defect and test responses to two alternative clinical strategies [65]. This approach allowed for a pre-emptive ex-vivo risk/efficacy assessment of available clinical strategies for management of cardiac disease in a complex patient, demonstrating the utility of hiPSCs for personalised medicine.

\section{MODELING DILATED CARDIOMYOPATHY: DMD CASE STUDY}

\section{Brief overview of DCM in DMD}

DCM is a prevalent cardiac manifestation in MDs with cardiac involvement (Table 1). It is characterized by progressive left ventricular dilation, fibrosis, and defects in CM contractile properties. Cardiac monitoring and management in the clinic are similar for all forms of MDs with DCM (reviewed in [4, 66]). Here, we will focus on DMD as a case study to illustrate advantages and challenges associated with hiPSCs in modelling DCM.

In DMD patients, cardiac disease is of early onset and by 20 years of age almost $100 \%$ of patients show cardiac involvement. Hor et al. studied 314 DMD patients using magnetic resonance imaging and detected cardiac fibrosis in $17 \%$ of patients $<10$ years of age and in $34 \%$ of patients $<15$ years of age [15]. As the disease progresses, fibrotic tissue gradually spreads and results in loss of contractility and DCM which is characterised by left ventricular (LV) dysfunction, thinning of LV wall, impaired diastolic and systolic function, leaky heart walls and often results in mitral valve regurgitation [67]. Markham and colleagues have shown signs of LV relaxation and decreased ventricular compliance via echocardiography in DMD patients <15years old [68]. Myocardial remodelling is a complex process that involves pathological cross-talk among various cell types, including fibroblasts, CMs, immune cells, and vascular cells. This process would be difficult to recapitulate in a dish with pure CMs. However, animal studies suggest that it is a secondary event to abnormalities that occur at the CM level (see below) and could therefore be captured in hiPSC cultures. 
In addition to tissue remodelling typical of DCM, cardiac involvement in DMD is also associated with ECG abnormalities that include increased R-to-S ratio $(\geq 1)$ in lead $\mathrm{V} 1$, tall $\mathrm{R}$ waves, deep $\mathrm{Q}$ waves in lead 1, arrhythmias, shorter PR interval and prolonged QT interval $[69,70]$. While no studies have been done so far on hiPSC-CMs from DMD patients that recapitulate these abnormal features in the dish, hiPSC-CMs have been used successfully to model long QT syndrome and arrhythmias in vitro [71-76]. Therefore, it is technically possible to study ECG abnormalities in cultured CMs from patient-derived hiPSCs.

\section{Molecular mechanisms of cardiac disease in $D M D$}

In DMD, frameshifting or nonsense mutations in the DMD gene cause loss of expression of a functional dystrophin protein in all striated muscles [77]. Dystrophin performs both structural and less well characterized signalling functions. The primary structural role of dystrophin is to link the intracellular sub-membranous actin network to laminin in the extracellular matrix via the transmembrane and extracellular members of the dystrophin-associated protein complex (DAPC). Loss of dystrophin expression in DMD, leads to a severe reduction of the DAPC at the muscle cell membrane. This disruption of the structural functions of the DAPC leads to membrane fragility in the face of repeated contractions and increased membrane permeability, accompanied by impaired calcium buffering and increased oxidative stress. These imbalances ultimately predispose muscle cells to necrosis. While this structural model is well validated in skeletal muscles, it does not completely fit with the current observations in cardiac muscle.

The structural components of the DAPC comprise dystrophin, $\alpha$ - and $\beta$-dystroglycans, $\alpha-, \beta-, \delta$-, and $\gamma$-sarcoglycans, and laminin [78]. Mutations in the genes encoding for these proteins invariably lead to MDs. Furthermore, glycosylation of $\alpha$-dystroglycan is essential for its binding to laminin and is critical for function. Thus, mutations in enzymes involved in the complex glycosylation of $\alpha$-dystroglycan are associated with MDs [79, 80]. If disruption in any of these proteins destabilizes the DAPC and compromises its structural functions, it would be expected that cardiac disease would be present in all related MDs and would show similar underlying molecular defects. However, this is not the case. In particular, mutations in laminin $\alpha 2$ cause severe skeletal muscle disease but are rarely associated with cardiac disease in MDC1A patients [81, 82]. If cardiac disease is present, it is usually mild. This observation could suggest that laminin (specifically laminin $\alpha 2$ ) is not the primary extracellular ligand of $\alpha$-dystroglycan in the heart. Other extracellular ligands have been identified for $\alpha$-dystroglycan. For most of them, binding appears to require $\alpha$-dystroglycan glycosylation [83]. It would therefore be expected that mutations in enzymes that glycosylate dystroglycan would disrupt multiple ligand interactions and therefore lead to DCM. However, among all identified enzymes that affect the glycosylation of $\alpha$-dystroglycan and lead to severe skeletal muscle disease, only mutations in two of them, Fukutin and Fukutin related protein, are associated with DCM (Table 1). This raises the interesting possibility that $\alpha$-dystroglycan binds to different extracellular matrix proteins in cardiac versus skeletal muscle with differential involvement of its core protein and carbohydrates moieties. How ligand binding affects the functions, structural or otherwise of $\alpha$-dystroglycan is currently unknown.

A second intriguing observation is that membrane integrity is not severely compromised in CMs in the dystrophin-deficient $m d x$ mouse. In contrast to skeletal muscle fibres, increased membrane permeability to vital dyes is primarily observed in $m d x$ CMs after strenuous exercise or challenge with adrenergic agonists $[84,85]$. Therefore, additional mechanisms must be in place to attenuate the damaging effects of the loss of dystrophin on membrane integrity in CMs, compared to skeletal muscle myofibres. This is supported by a growing body of evidence indicating progressive intracellular elevation of calcium in dystrophin-deficient CMs via perturbation in ion channel function, rather than a catastrophic rise in intracellular calcium associated with membrane tearing [86-88]. Finally, in MDs associated with mutations in $\beta$ - or $\delta$-sarcoglycans, mechanisms extrinsic to CMs have been shown to contribute to DCM in mouse models [89]. These studies have implicated smooth muscle defects leading to arterial constrictions that cause regional ischemia as contributors to CM death. A similar pathological mechanism is not observed in DMD and associated animal models. Therefore, although many of the MDs listed in Table 1 involve proteins that are part of the DAPC, DCM does not necessarily arise from identical underlying molecular and cellular defects, with potential implication for treatment responses. 
Furthermore, experiments by our group have recently revealed key differences in the protein composition of the DAPC between cardiac and skeletal muscles in the mouse [90]. Interestingly, we reported that most differences involve the intracellular binding partners of dystrophin, $\alpha$-dystrobrevin, syntrophins, and neuronal nitric oxide synthase (nNOS). These proteins are involved in the less characterized signalling functions of dystrophin; these include mechanotransduction, nitric oxide signalling, clustering of membrane and extracellular matrix proteins, and regulation of ion channels, calcium homeostasis, cell survival, and membrane targeting of proteins [91-99]. We also reported novel cardiacspecific associations of dystrophin with four proteins involved in cardiac disease: Cavin-1 (PTRF), $\alpha$-Bcrystallin, Cypher (LDB3, ZASP) and Ahnak1 [90]. The functional significance of these differences in DAPC composition between cardiac and skeletal muscles is currently under investigation.

Taken together, these observations highlight gaps in our knowledge of the molecular underpinnings of cardiac disease in DMD and in related MDs involving the DAPC. Modelling these diseases in a dish using patient-derived hiPSCs offers unique opportunities to contrast and compare disease triggers in CMs. This is critically important to determine whether DCM associated with different DAPC-related mutations is truly one disease and one treatment can fit all.

\section{What disease parameters can be studied in hiPSC-CMs?}

\section{Developmental progression of disease} and correlation with genetic mutation

The DMD gene is extremely complex. It contains 7 different promoters with tissue-specific and developmentally regulated expression patterns. They encode 3 full length transcripts that contain all 79 exons leading to $420 \mathrm{kDa}$ proteins, as well as shorter protein isoforms with different $\mathrm{N}$-termini that vary in size from $260 \mathrm{kDa}(\mathrm{Dp} 260)$ to $71 \mathrm{kDa}(\mathrm{Dp} 71)$. The functional significance of this complex pattern of isoform expression and switching during maturation is not well understood. Differentiation of hESCs into CMs was used to show that isoform Dp71b was expressed in both undifferentiated and differentiated cells, whereas Dp140 and the long muscle-specific isoform, Dp427m, were expressed only upon the onset of CM differentiation [100]. These studies suggest that multiple dystrophin isoforms are expressed during maturation of human $\mathrm{CMs}$, although these findings need to be confirmed in a more homogeneous culture system. The possible implication is that depending on the location of the mutation along the $D M D$ gene, it could abrogate expression of one or multiple dystrophin isoforms, with potentially different consequences for $\mathrm{CM}$ maturation and function. The availability of newer monolayer differentiation protocols that produce high purity hiPSC-CMs and the demonstration that specific $\mathrm{CM}$ subtypes can now be produced will allow a better understanding the developmental expression of dystrophin isoforms [21]. This opens the door to detailed studies of the effects of different mutations along the DMD gene on $\mathrm{CM}$ ultrastructure and contractile parameters.

\section{Membrane fragility and calcium handling}

Of the functional consequences of the muscular dystrophies, calcium handling is best studied in hPSC-derivatives. Aberrant handling in DMD is typified by increased intracellular calcium and abnormal calcium sparks, particularly in response to contraction and stress [87, 101-103]. The underlying causes have not been elucidated and mechanisms may differ between different forms of MDs. In DMD, two hypotheses have been advanced with respect to the cause of increased intracellular calcium. The first postulates that loss of dystrophin causes membrane fragility leading to membrane tears that facilitate calcium influx. However, there is no direct evidence to support this hypothesis in the heart. The best indirect evidence is the observation that membrane sealants such as Poloxamer P188 can normalize intracellular calcium levels in dystrophin-deficient mouse cardiac myocytes [53]. The second proposes that loss of dystrophin disrupts either directly or indirectly the regulation of ion channels that regulate calcium homeostasis in the heart. In support of this hypothesis, elevated intracellular calcium has been reported in resting CMs and appears to be in part associated with increased expression of stretch activated receptors [102].

DMD hiPSC-CMs could be extremely useful in testing the relative contribution of these two different hypotheses to elevated intracellular calcium during differentiation and CM maturation. Past studies give credence to the potential use of hiPSC-CMs in such an investigation. Models using hiPSC-CMs have been produced for LQTS8 / Timothy Syndrome (mutations in the $\mathrm{I}_{\mathrm{Ca}-\mathrm{L}}$ calcium channel; [104] and catecholaminergic polymorphic ventricular tachycardia-1 (CPVT1; mutations in ryanodine receptor; RYR2; [71, 105, 106]. In CPVT1 
hiPSC-CMs, dantrolene was shown to abolish isoprenaline-induced arrhythmias [71]. In addition, the use of calcium assays in hiPSC-CMs is becoming commonplace due to the ease and availability of high speed / resolution optical mapping techniques. Typically, this uses voltage-sensitive dyes (e.g. ANEPPS) [107] or genetically-encoded voltage indicators [108] to measure action potentials and calcium wave propagation. Indeed, Guan and coworkers showed a two-fold increase in $\mathrm{T}_{50}$ (duration of recovery) of $\mathrm{Ca}^{2+}$ transients in DMD hiPSCCMs compared to healthy hiPSC-CMs. Although this is in agreement with data from other model systems investigating skeletal muscle [109-111], only one DMD hiPSC line was studied and a more detail investigation is needed. In this regard, Lin and co-workers examined a collection of DMD, BMD (Becker Muscular Dystrophy) and healthy hiPSCCMs. This included demonstration that treatment for 7 days with the membrane sealant, Poloxamer P188, could reduce both caspase- 3 activation and apoptosis in the DMD hiPSC-CMs. In particular, staining of the treated DMD hiPSC-CMs with the $\mathrm{Ca}^{2+}$ sensitive dye, Rhod-2AM, revealed increased cytosolic $\left[\mathrm{Ca}^{2+}\right]_{\mathrm{i}}$ concentration [53].

\section{Mitochondrial function}

The overload in calcium that is associated with muscular dystrophies can lead to compromised mitochondrial function [112]. The utility of evaluating mitochondrial function using the Seahorse XF Flux analyser has been demonstrated in several hiPSC-CM models of disease, including Barth syndrome [60], Diabetes type I [113] and ARVD [63]. For example, in Barth syndrome, hiPSC-CMs demonstrated reduced ATP production and respiration capacity [60] when exposed to sequential treatment with electron transport chain inhibitors such as oligomycin, antimycin-A and rotenone and FCCP. However, using DMD hiPSC-CMs, Guan and colleagues did not find any differences in mitochondrial metabolic function [111], which is in contrast to other studies. Thus, in diseased hiPSC-CMs, swollen mitochondria were observed using transmission electron microscopy, while staining with the potentiometric dye, JC-1, showed a $\sim 2.5$-fold increase in the number of mitochondria with disrupted membrane potential [53]. Other reports show reduced oxidative phosphorylation in vitro, in vivo and in isolated mitochondria from skeletal muscles of mdx mice or DMD patients [114, 115]. Further work is needed to understand the cause of these differences.

\section{Electrophysiology / E-C coupling}

hiPSC-CMs have been extensively used to recapitulate and study ex vivo cardiac disorders associated with conduction defects or electrocardiogram (ECG) abnormalities such as long QT syndrome (Reviewed in [116]). Importantly, these studies have demonstrated a strong correlation between results obtained with hiPSC-CMs and primary adult human CMs. Furthermore, they have shown that patient-derived hiPSC-CMs can be used to predict in vivo responses of the whole heart to drug treatments.

Currently, a variety of techniques are available to study electrophysiology in cultured CMs ranging from field action potentials to patch clamp recordings on individual ion channels (reviewed in [55]). Abnormal ion channel function has been reported in primary CMs from DMD mouse models. This has been evidence by reduced inactivation kinetics of Ltype calcium channels and over-activation of stretch activated channels in mouse models of DMD resulting in increased intracellular calcium concentrations and affecting action potential kinetics [87, 102]. In addition, direct and indirect mechanisms that affect $\alpha$-syntrophin and alternative splicing of $\alpha$ dystrobrevin give rise to abnormal regulation of sodium channels in myotonic dystrophy and are associated with long QT syndrome [117-119].

In addition to direct measurement of membrane voltage, quantification of calcium sparks and intracellular calcium levels in cultured CMs is possible. Increased intracellular calcium and abnormal calcium sparks have been reported in CMs from animal models of neuromuscular disorders both in response to contraction and following stress $[87,102,103$, $120,121]$. Increased intracellular calcium has been linked to mitochondrial dysfunction and structural abnormalities in dystrophic CMs, predisposing to apoptotic cell death. To date, limited studies have been performed on hiPSC-CMs from DMD patients (Table 2). The current data show impaired calcium handling similar to CMs derived from animal models and indicate a similar role in impaired mitochondrial function and apoptosis [53, 111]. These early studies also demonstrate that hiPSCs derived from DMD patients can generate atrial, ventricular and nodal CMs [111]. This offers the possibility to study the effects of underlying genetic mutations on the electrophysiological properties of each of these CM sub-types at a level of cellular and sub-cellular resolution that cannot be achieved in vivo. Therefore, hiPSCs represent a powerful tool to model conduction and ECG defects associated with MDs at the 


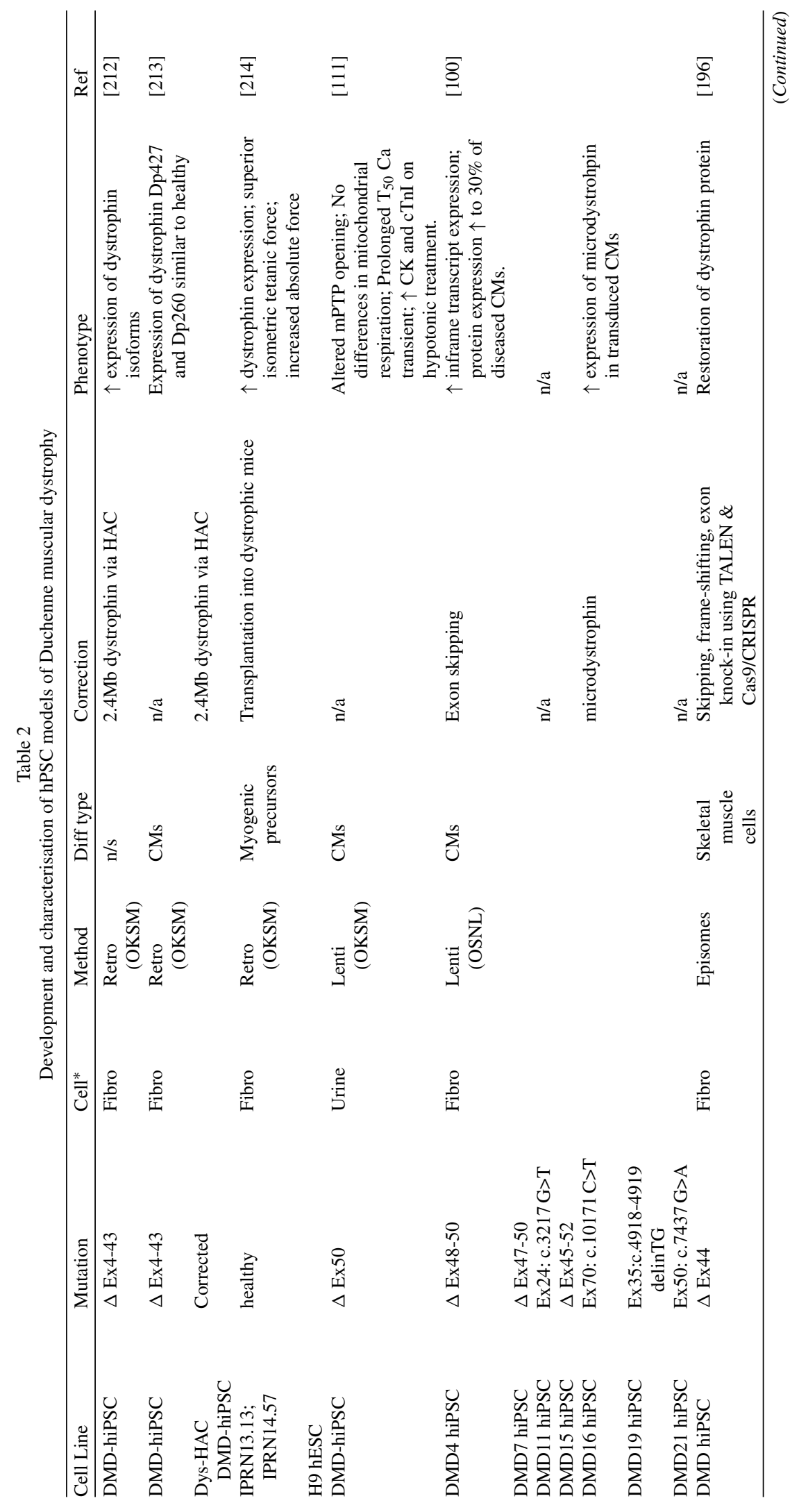




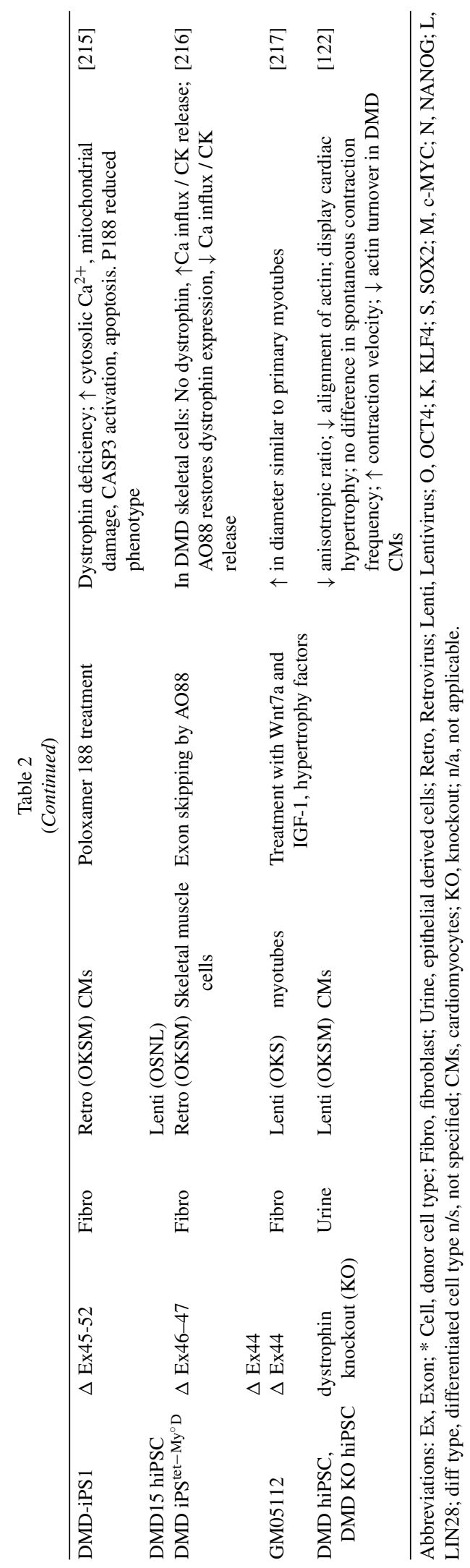


level of the individual patient. Future use of these cells to assess electrophysiology will be informative in a system that more accurately reflects the complexity of protein splice variants, the heterogeneity of underlying genetic mutations and the electrophysiological properties of the human heart compared to animal models.

\section{Structural/sarcomeric abnormalities}

Imaging has also been used to investigate differences between healthy hiPSC-CMs relative to DMD hiPSC-CMs or engineered DMD knock out hiPSC-CMs. Whereas healthy hiPSC-CMs have an aspect ratio (length: width) of $\sim 5$, the value is $\sim 3.8$ for dystrophic hiPSC-CMs. The dystrophic CMs also showed significant increase in cell surface area compared to healthy CMs, which corresponded to reduced actin turnover. These observations may explain the reduced ability of dystrophic hiPSC-CMs to respond to environmental cues, including the nanotopography of the substrate [122].

\section{Myocardial contractility and function}

There are several aspects of myocardial dysfunction that are commonly observed in MDs. At the level of the whole myocardium, deficits in force production, a blunted response to adrenergic stimulation, and progressive ventricular dilation are commonly observed. Only relatively recently has contraction force been measured in hiPSC-CMs. This shows that twitch forces are in the $2-260 \mathrm{nN}$ range for single hiPSC-CMs [123-125] and $0.08-4.4 \mathrm{mN} / \mathrm{mm}^{2}$ range for 3D engineered heart tissue (EHTs) derived from hiPSC-CMs [126]. The impact of different drugs on hiPSC-CM contractility has also been measured. Impedance-based have been used to test a panel of 49 cardioactive compounds on hiPSC-CM. The sensitivity, specificity and accuracy was reported as $90 \%, 74 \%$, and $82 \%$, respectively, which compared favourably when compared to in vitro data for dog $(83 \%, 84 \%$ and $82 \%)$ and rat $(77 \%, 74 \%$, and $74 \%)$ CMs [127]. EHTs made from rat, mouse, and human CMs have differences in the maximal twitch forces $(0.22,0.05$, and $0.08 \mathrm{mN})$ and sensitivity to external $\mathrm{Ca}^{2+}\left(\mathrm{EC} 50: 0.15,0.39\right.$, and $\left.1.05 \mathrm{mM} \mathrm{Ca}^{2+}\right)$ [128].

DMD hiPSC-CMs carrying an exon 45 deletion (described previously [111]) and isogenic DMD knockout hiPSC-CMs have high contraction velocity of $10-20 \mu \mathrm{m} / \mathrm{s}$ relative to $5-7 \mu \mathrm{m} / \mathrm{s}$ in healthy CMs. Furthermore, healthy hiPSC-CMs on nano-patterns showed greater anisotropic contractions whereas high variability in contraction direction was seen in DMD hiPSC-CMs [122].

\section{MODELING CONDUCTION SYSTEM DEFECTS (CSDS): DM1 CASE STUDY}

\section{Brief overview of cardiac disease with CSDs}

CSDs are a predominant feature of cardiac disease in myotonic dystrophies and in MDs with mutations in nuclear proteins (e.g. Emery-Dreyfuss muscular dystrophies; limb-girdle muscular dystrophy 1B; Table 1). CSDs can occur alone or in association with DCM. In general, management involves pharmacological and device based (implantation of pacemakers or defibrillators) interventions (reviewed in [66, 129]). DM1 cardiac pathology commonly involves tachyarrhythmias and defects in conduction systems due to degeneration, fibrosis and fatty infiltration, and, less often, myocardial dysfunction and ischemia. Conduction abnormality is mainly characterised by defects in sinus node, atrio-ventricular node and His-Purkinje system, and is mostly associated with prolonged PR interval, QRS duration and His bundle to ventricle (HV) interval [130]. 65\% of DM1 patients exhibit abnormal ECG patterns, which include first degree AV block (42\%), right (3\%) and left (4\%) bundle branch block respectively and intraventricular conduction delay (12\%) [131]. DM1 patients also suffer from risk of developing bundle branch tachycardia and atrial arrhythmias, fibrillation and flutter, are common. Echocardiographic abnormalities include mitral valve prolapse, systolic and diastolic function impairment, and reduction in ejection fraction [130]. Current cardiac management includes pharmacological interventions and device implantation [129].

\section{Current knowledge of disease mechanisms}

DM1 is due to unstable expansion of CTG trinucleotides in 3'-UTR of the DMPK gene [132], which is considered to form a stem loop regulated by RNA binding proteins. Numerous mechanisms that create diverse phenotypic effects have been previously proposed [133]. In brief, accumulation of expanded mRNA in DM1 cells, also referred to as foci, is considered as hallmark feature of DM1, which mediate RNA gain-of function. Accumulating foci cause muscleblind-like (MBNL) protein sequestration, as well as concurrent activation and increased expression of CELF1 [134, 135]. Both MBNL and CELF1 
regulate splicing, with aberrant function altering profile of cardiac troponin $\mathrm{T}$, muscle-specific chloride channel and sarcoplasmic/endoplasmic reticulum $\mathrm{Ca}^{2+}$ ATPase1 [136-138]. Alternatively, depletion of MBNL has shown to perturb (i) alternative polyadenylation in mouse embryo fibroblasts, skeletal muscle in transgenic mouse DM1 model and DM1 patients; (ii) regulation of mRNA localisation; (iii) regulation of mRNA transcription and translation [139, 140]. MBNL1 may also regulate miR-1 microRNA biogenesis, acting via the LIN28 pathway, which could cause aberration of calcium channels and gap junctions, hence explain some of the abnormal cardiac function seen in DM1 patients [141]. Mutant RNA repeats are also suggested to play role in other RNA binding proteins such as Staufen1 and Dead box RNA helicases, which regulate protein translation [142, 143]. Additional hypotheses exist which supports the role of expanded CTG repeats in triggering hypermethylation of rRNA promoters, inhibition of rRNA transcription [144], and disruption of other cellular processes including repeats associated nonATG (RAN) translation [145] and antisense DMPK transcription [146, 147].

\section{What disease parameters can be studied in hiPSC-CMs?}

\section{Developmental progression}

A high degree of repeat instability can occur between different somatic tissues and during transmission from DM1 parents to children, a phenomenon known as genetic anticipation. However, the mechanism(s) by which this occurs are limited (reviewed in [148-150]) and DM1 hPSC models could provide insights. Several reports have shown instability during the reprogramming process or during culture of DM1 hPSCs but not in differentiated cells, including osteogenic progenitor cells [151-153]. Nevertheless, these observations contradict in vivo data where CTG repeats are unstable during oocyte development but stable in zygote and embryo $[154,155]$. Additionally, no repeat instability was observed in the 13 week-old human DM1 foetus, although heterogeneity did occur by 16 weeks of gestation [156, 157]. The reason for these differences will need to be resolved before the value of DM1 hiPSCs can be determined but may relate to differences in species or (epi) genetics. Other influencing parameters such as starting repeat length or differentiated cell types assessed could be evaluated using the hiPSC model, particularly when compared to corrected isogenic controls produced by gene targeting. Developing such data would provide the foundation with which to evaluate the ability of new therapeutics to inhibit repeat expansion.

\section{Understanding and restoring gene function}

Understanding gene function and evaluating the impact of potential therapeutic interventions is the area of research that has received the most attention in the DMD and DM1 hPSC field (Tables $2 \& 3$ ). For example, DM1 is characterised by improper gene expression and methylation. Yanovsky-Dagan and co-workers studied 14 different DM1-hESC lines and showed that hypermethylation occurred upstream of CTG repeats when repeat number exceeded 300 [158]. The process of reprogramming patient fibroblasts into hiPSCs resulted in hypermethylation but was not associated with an expansion in the repeat size. The authors of that study also went on to show that there was a negative correlation between methylation and expression of neighbouring gene, SIX 5, but no correlation of $D M P K$ expression in DM1 hESCs and DM1 hESC-CMs [158].

The RNA gain of function mechanism in DM1 suggests that CUG repeats induce symptoms of DM1 by altering the function of CUG-binding splice regulating proteins, including MBNL1 and CUGBP1. The over-expression of human CUGBP1 adult mouse heart has shown to reproduce functional and molecular abnormalities of DM1 [159]. Recently, cardiac phenotypes and MBNL sequestration has been studied using a Drosophila model expressing expanded repeats under cardiac-specific promoter GMH5-Gal4 [160]. So far, such events have only been investigated in non-CM lineages from hPSCs (Table 3). $\mathrm{Xia}$ and co-workers showed presence of RNA foci in cells from all three germ layers derived from DM1-hiPSCs [161]. Similarly, PGD-derived DM1hESC lines [162] differentiated into neural stem cells showed formation of RNA foci along with decreased proliferation and increased autophagy. This model also offered the opportunity to evaluate potential genetic interventions, both to shed mechanistic insight to DM1 and to evaluate potential treatments. Thus, involvement of MBNL1 in mTOR signalling was demonstrated by siRNA knockdown [163], while a targeted approach positioned poly A signals upstream of CTG repeats in the DMPK gene by TALEN-mediated genome engineering in DM1 hiPSC-derived neural stem cells. The latter prevented production of CUG expanded transcripts and restored healthy phenotype, as gauged by loss of RNA 


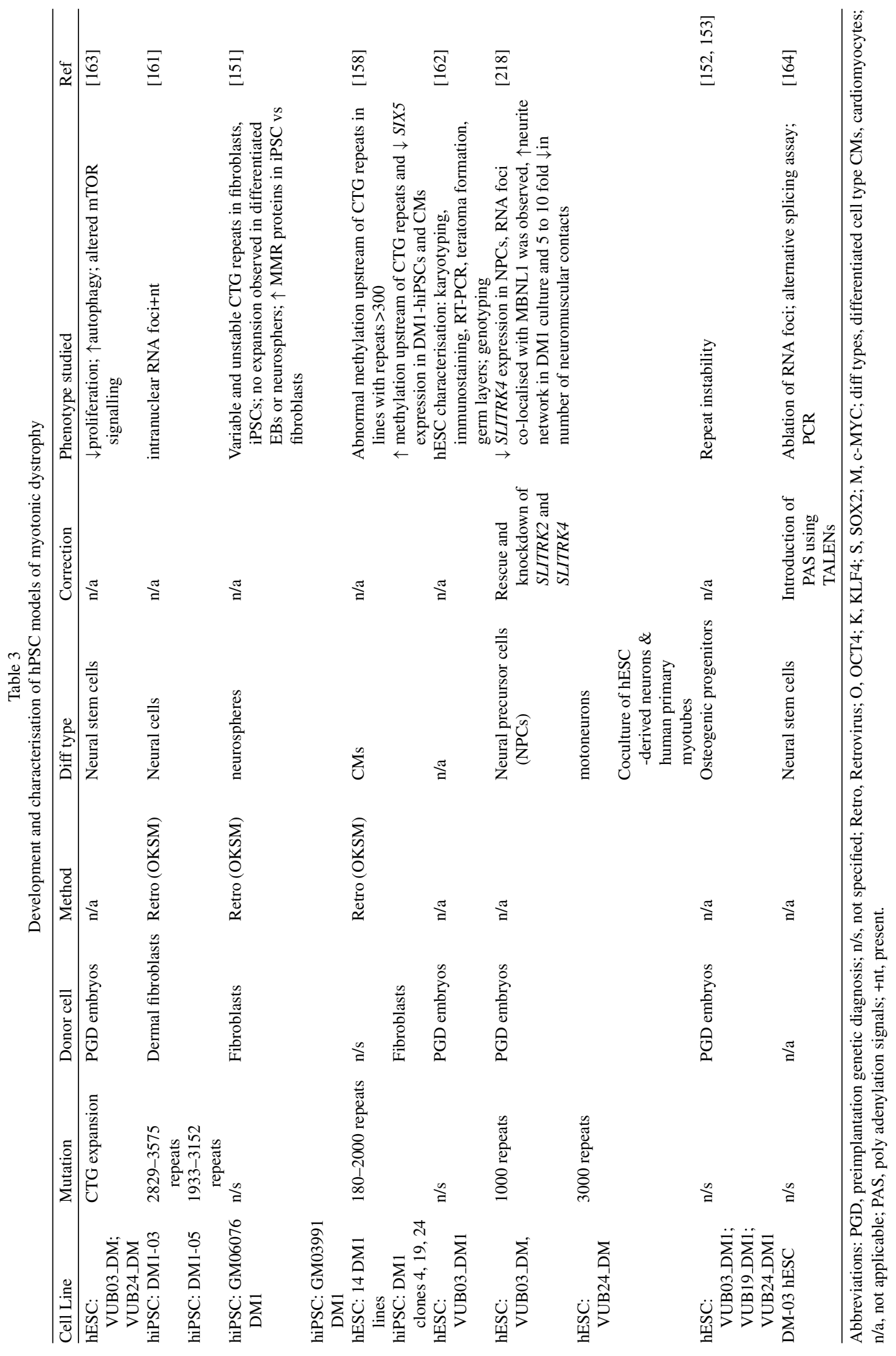


foci, increased expression of microtubule-associated protein tau (MAPT) and decreased expression of MBNL1 \& 2 [164]. Nevertheless, TALENs targeted both the healthy and mutated allele, whereas a therapeutic would have to modify only the mutated DM1 allele [164].

Small molecules are also being considered as potential therapeutics for reduction of RNA foci in DM1. A medium throughput phenotypic assay in patient-derived fibroblast and myoblast cells was used to screen a total of $\sim 16,000$ compounds from a Chembridge Diverset, NPC and Enzo Life Sciences libraries. This identified the protein kinase $\mathrm{C}$ inhibitor, Ro 31-8220, as being able to reduce and/or remove nuclear foci [165]. For hiPSC-CMs, imaging and particularly high content imaging is now becoming commonplace to examine subcellular structures, which will be important to investigate the resolution of RNA foci in DM1. In addition to validating the efficacy of drugs such as Ro 31-8220, the hiPSC-CM system will be useful in evaluating potential cardiotoxicity, which is well established issue associated with kinase inhibitors [166]. Thus, Mioulane and co-workers [167] triggered cell stress with the cellpermeable protein kinase $\mathrm{C}$ inhibitor, chelerythrine. Combining stains for TMRM (a potentiometric dye; tetramethylrhodamine methyl ester), caspase-3 or BOBO-1 with Cellomics Arrayscan imaging, they assessed mitochondrial function, apoptosis and cell death, respectively and demonstrated that hPSC-CMs are less susceptible to chelerythine-stimulated apoptosis compared to rat neonatal ventricular CMs. In an impressive study, Sirenko and colleagues [168] used CalceinAM, Hoechst and MitoTracker imaging on the ImageXpress Micro to evaluate cardiotoxicity of 131 modulators of $\mathrm{Na}^{+}, \mathrm{K}^{+}$and $\mathrm{Ca}^{2+}$ channels, as well as adreno-, dopamine- and histamine-receptors on CDI iCell hiPSC-CMs in a 384-well format.

\section{Calcium handling}

Several studies have reported altered $\mathrm{Ca}^{2+}$ handling in DM1 muscles, DM1 myotubes and DM1 mouse models [169-171]. More recently, aberrant splicing affecting a number of channels (such as SERCA1, RyR1 and CACN1S) is thought to be involved in altered $\mathrm{Ca}^{2+}$ signalling in DM1-affected muscles. For example, exon 29 of the CACN1S gene is abnormally skipped in DM1 patients. Inducing exon 29 skipping in wild type mice leads to increased $\mathrm{Ca}^{2+}$ channel conductance and voltage sensitivity, thus affecting EC coupling [172]. Enhanced skipping of CACN1S exon 29 in the DM1 mouse model exacerbates muscle pathology and better mirrors disease severity seen in DM1 patients [172]. The combined effect of mis-spliced SERCA1, RyRl and $C A C N 1 S$ genes is increased EC coupling, as well as altered $\mathrm{Ca}^{2+}$ release and uptake from SR. This is likely to cause chronic $\mathrm{Ca}^{2+}$ accumulation, a common phenomenon thought to occur in MD. More recent studies using microarrays on DM1 patients identified $\mathrm{Ca}^{2+}$ signalling as the most significantly affected pathway, showing upregulation of $14 \%$ of genes involved in $\mathrm{Ca}^{2+}$ release, uptake, storage, or signalling [173]. Differential splicing of CACN1S has been demonstrated in DM1 mouse and human models. However, the difference could be attributed to various factors including species difference in splice regulation, difference in repeat expansion in models studied or difference in RNA flanking the CTG repeat [172]. Some of the listed limitations can be overcome by using isogenic hiPSC lines to study the mutation-specific effect in a genetically controlled environment.

\section{Mitochondrial function}

In humans, four out of seven DMPK isoforms anchor into the outer mitochondrial membrane or endoplasmic reticulum, indicating a role for DMPK in mitochondrial dysfunction. Elevated expression levels of the mutated DMPKA isoform is implicated in numerous cell changes, as determined from studies using a range of cell lines, and heart and skeletal muscle of transgenic mice. These changes include perinuclear clustering, abnormal mitochondrial ultrastructure, decreased mitochondrial membrane potential and release of cytochrome C, which lead to apoptosis [174, 175]. Recently it has been reported that alternative splicing causes increased expression of pyruvate kinase M2 in DM1 skeletal and heart tissue. This results in increased glucose consumption but decreased oxidative phosphorylation, suggesting that the energy production is less efficient in DM1 cells [176]. Mitochondrial function studies have already been successful in hiPSCs derived from Barth syndrome [60] and diabetic patients [113], which gives confidence that in vivo changes in bioenergetics could be recapitulated in DM1 hiPSC-CMs.

\section{Electrophysiology/EC coupling and contractility}

With the increased awareness of cardiac involvement in muscular dystrophies, there is a concomitant increase in publications on Holter, ECG and MRI studies. These show that $90 \%$ of DMD patients show 
cardiac involvement as the disease progresses. In the case of DM1, a pan-European registry identified the PR interval of $29 \%$ patients was $>200$ msecs on the electrocardiogram, while $46 \%$ experienced arrhythmia or conduction block, of which $37 \%$ had a cardiac implant (pacemaker or implantable cardioverter defibrillator) [177].

For DM1, cardiac conduction defects and altered contractility have been observed in $\mathrm{DMPK}^{-/-}$ knockout mice and isolated human primary DM1 CMs [178-180], which parallels the observations made in the hearts of DM1 patients. More recently, prevalence of Brugada type 1 ECG pattern have been reported in DM1 patients, implicating the role of Brugada syndrome in tachyarrhythmias and cardiac death $[181,182]$. The cardiac electrical and contractile phenotype of DM1 has been studied using a mouse model; this constitutively expresses the human DM1 locus under the regulation of its own promoter and cis-regulatory elements, after flecainide treatment [183]. The altered $\mathrm{I}_{\mathrm{Na}}$ in this mouse model was suggested to be the cause of reduced cellular excitability [183]. Furthermore, function and localisation of $\mathrm{Cl}^{-}$ channel (PLM) is also modulated by DMPK, suggesting that DMPK is involved in EC coupling in muscle cells [184]. The loss of DMPK in knockout mice has been shown to enhance the basal contractility of single CMs concurrent with an associated increase in intracellular calcium during systole, suggesting role of DMPK in control of calcium homeostasis [120]. Furthermore, reduced expression of miR-1 microRNA in DM1 patient hearts has been reported to alter the regulation of connexion 43 and CACNA1C, which may contribute to the cardiac abnormalities observed in affected patients [141]. Although, these parameters have not yet been examined in DM1 hiPSC-CMs, they have been successfully studied in other cardiac diseases as discussed above (see section on electrophysiology and myocardial contractility in DMD).

\section{OPPORTUNITIES AND CHALLENGES}

In recent years there has been considerable progress in the culture, differentiation technologies and genetic modifications associated with hiPSCCMs. Nevertheless, this is still a nascent technology and there are still considerable challenges to be overcome. There is a need for: 1) standardisation of reprogramming methods (viral vs non-viral/ integrating vs non-integrating) as it can affect the genome and epigenome; 2) introduction of standards on how to select good quality cell lines that should be used for screening and disease modelling; 3 ) determination of the number of cell lines per patient that need to be generated to control for variations arising from differences in reprogramming; 4) optimization of protocols to improve reproducibility and robustness of cardiac differentiation in order to allow translation from basic research laboratories to industrial and biomedical platforms.

Limited maturation occurs in hiPSC-CMs, which is consistent with mid-gestation of human foetal heart development. This is evidenced by examining 30 or so parameters that are used to quantify $\mathrm{CMs}$, including structure, gene expression, amount of sarcoplasmic reticulum, metabolism, mitochondria location and number, electrophysiology, calcium handling and contractility [21]. Thus, while hiPSC$\mathrm{CMs}$ are functional and already have proven utility in drug and disease valuation, all phenotypes might not be fully recapitulated. Thus, there is not only a need for careful interpretation of data relative to appropriate (e.g. isogenic) controls, but also an appreciation of the current limitations of the model. For example, immature hiPSC-CMs lack well-developed T-tubule system and show action potential and calcium parameters similar to embryonic heart, making it difficult to study the distribution and function of dystrophin in hiPSC-CMs. We have previously shown a diffused pattern of dystrophin in hiPSC-CMs [100], which contrasts to the defined membrane localisation pattern in adult skeletal and heart muscle [185]. Clarity will also be required on details such as whether all the components of DAPC are present, correctly localised and functional in immature CMs. Naturally there are major efforts to address maturity in the knowledge that each incremental improvement could make a considerable difference to the utility of the models produced. Indeed, many investigators are evaluating physical, chemical, genetic and environmental inducers to facilitate maturation (reviewed in detail in [21, $186,187])$. It will be interesting to see if the abnormal expression of embryonic splicing of $M B N L$ and $C U G B P$ (discussed above) is maintained in DM1 hiPSC-CMs and if maturation of CMs has any effect on this.

Although large scale differentiation can produce hiPSC-CMs at purities of $>80 \%$, the cultures are heterogeneous with a mixture of ventricular, atrial and pacemaker subtypes. The quality of data produced would likely be enhanced if single subtypes could be isolated. Until recently, reliable methods to 
isolate subtypes remained elusive. However, there have been recent exciting developments. Birket and colleagues [188] combined a complex but elegant double transgenic approach, wherein an NKX2.5GFP targeted hESC line was further transfected with an inducible MYC expression construct. In the presence of insulin-like growth factor-1 (IGF-1) and a hedgehog pathway agonist, cardiovascular progenitor cells could be isolated and proliferated for over 40 population doublings. Moreover, modulating exogenous BMP, FGF, WNT and RA signalling led to multi-lineage differentiation, as well as directed specification to pacemaker and ventricular cells. This report was remarkable because it not only showed long-term proliferation of hPSC-derived cardiac progenitors (in 11 other reports using mouse and human PSCs, maximum expansion was 4-fold) [189], but it was the first robust demonstration of subtype specification. In an alternative approach, modulation of retinoic acid signalling during $\mathrm{hESC}$ differentiation was used to generate atrial- and ventricular-like CMs. These CM subtypes were used to show that the multiion channel blocker, vernakalant, and Kv1.5 blocker, XEN-D0101, caused a reduction in early repolarization only in the atrial cells [190], providing a novel preclinical test platform for these drug classes.

As explained above, the breath of approaches to phenotype cell is expanding with a greater reliance on high content platforms, which will increase the throughput available. However, to confirm the phenotype and evaluate the significance of what is observed in culture, parallel studies in vivo are important, but lacking. The DMD and DM1 hiPSC-CM models that have been generated so far have predominantly relied on cells cultured in 2D on tissue culture plastic. This represents a rather simple model compared to the complex 3D architecture of the intact heart, wherein there is a combination of CMs, cardiac fibroblasts, endothelial cells and smooth muscle cells that experience continuous contractile and haemodynamic forces. It has already been shown that combining hiPSC-CMs with vascular and/or fibroblastic cells can improve function [191, 192]. Further work is needed to determine whether engineering complex tissue and organ models can improve the hiPSC-CM models to create unique disease models for studying multifaceted phenotypes in certain MD.

The potential of permanent genetic correction is rising to the fore with the advent of nucleasemediated gene targeting approaches and proof of principle studies have been conducted in hPSC models [193-197]. For example, TALENs have been shown to allow correction of DMD hiPSC-derived skeletal muscle cells carrying deletion in exon 44 via frameshift, exon skipping and exon knock-in approaches [196]. In other DMD studies, TALENs and ZFNs have been shown to enhance expression of dystrophin by correcting the mutation ( $\Delta$ exon 48-50) in DMD-derived myoblasts by correcting the reading frames [198, 199]. TALEN-mediated genome engineering has enabled poly A signals to be positioned upstream of CTG repeats in DM1 hiPSCderived neural stem cells to prevent expression of mutant $D M P K$ transcripts. However, there are no reports of direct correction of the $D M P K$ gene in DM1 hPSCs. This is likely to be due to the highly repetitive and complex nature of the repeat region, which may interfere with the targeting strategy. Such difficulties may be overcome with high efficiency Cas9/CRISPR approaches.

More controversially, genome engineering raises the possibility of correcting mutations in the germline, fertilised egg or early stage embryo and the first attempts to use this technology have been made. The Cas9/CRISPR system was used in a somewhat abortive and controversial [200] attempt to target the $\beta$-globin gene in human embryos. However, the efficiency of targeting was low (14\%), edited embryos were mosaic and the level of off-target events was high due to homology with delta-globin gene [201]. Earlier in Feb 2016, the first gene editing study in human embryos has been approved and whether such studies will more widely be given approval and if these will be suitable for correction of DMD and DM1 mutations remains to be seen. If they are, it is likely small polymorphisms of a few bases that cause premature termination would be targeted first due to the complexity of correcting multi-intron deletions in DMD or highly repetitive triplet repeats in DM1.

\section{CONCLUDING REMARKS}

The ability to produce hiPSC-CMs offers new opportunities in biomedicine. Over the last 5 years a swathe of hiPSC-CM models have been developed for genetic conditions that affect the electrophysiology, signalling and survival of cells within the heart. Whereas a few years ago, evaluating a few drugs for cardiotoxicity was a major undertaking, improvements in the hiPSC-CM technology mean single reports can now screen over 130 compounds, showing acceleration in the field. Many major academic institutes and pharmaceutical companies are now 
evaluating hiPSC-CMs, with excellent outcomes: Refinement in patient risk stratification for sudden cardiac death; progression of anticancer drugs to the clinic; the first clinical trials for heart failure. Such developments are now percolating into the muscular dystrophy field with 5 of the 10 DMD and 1 of the 7 DM1 models investigating hiPSC-CM function. In some cases, these have been used to evaluate corrective therapies. Nevertheless, this is a nascent field. Alignment of large scale hiPSC banking initiatives with pan-EU initiatives (e.g. MRC BioBank, EuroBioBank network, TREAT-NMD Network of Excellence) for rare diseases, including DMD and DM1, is only just starting. The potential of using Cas9/CRISPR genome engineering technologies to exponentially increase the diversity of mutations and genotypes available is only now becoming reality but may provide a way to unveil the impact on function of single nucleotide polymorphisms identified via genome-wide association studies (GWAS). Such studies will require methods that can assess hiPSC$\mathrm{CM}$ function in detail, whilst being sufficiently high throughput to examine phenotype across large numbers of hiPSC lines or genome engineered derivatives. As these tools are developed, there should be better correlation of clinical pathology with in vitro phenotype and hence evaluation of potential therapies. This should also help resolve debates on whether treatments that have shown benefit to skeletal muscle have a positive or negative impact in the heart. Realising these goals will require better understanding of the biology of hiPSC-CM maturation and subtype specification to make the models more representative of the working heart. For each of these areas, international efforts are underway and it can be expected that over the next 5 years hiPSC-CMs will become an important additional tool in muscular dystrophy research and therapy.

\section{REFERENCES}

[1] Flanigan KM. The muscular dystrophies. In Seminars in Neurology. 2012.

[2] McNally EM. New approaches in the therapy of cardiomyopathy in muscular dystrophy. Annu Rev Med. 2007;58:75-88.

[3] Gagnon C, et al. Predictors of disrupted social participation in myotonic dystrophy type 1 . Archives of Physical Medicine and Rehabilitation. 2008;89(7):1246-55.

[4] Bushby K, Muntoni F, Bourke JP. 107th ENMC international workshop: The management of cardiac involvement in muscular dystrophy and myotonic dystrophy. 7th9th June 2002, Naarden, the Netherlands. Neuromuscul Disord. 2003;13(2):166-72.
[5] Limongelli G, et al. Skeletal muscle involvement in cardiomyopathies. J Cardiovasc Med (Hagerstown). 2013; 14(12):837-61.

[6] Kaspar RW, et al. Analysis of dystrophin deletion mutations predicts age of cardiomyopathy onset in becker muscular dystrophy. Circ Cardiovasc Genet. 2009;2(6): 544-51.

[7] Nicolas A, et al. Becker muscular dystrophy severity is linked to the structure of dystrophin. Hum Mol Genet. 2014.

[8] Heydemann A, Wheeler MT, McNally EM. Cardiomyopathy in animal models of muscular dystrophy. Curr Opin Cardiol. 2001;16(3):211-7.

[9] McNally E, et al. Cytoskeletal defects in cardiomyopathy. J Mol Cell Cardiol. 2003;35(3):231-41.

[10] Yu X, et al. Dystrophin-deficient large animal models: Translational research and exon skipping. Am J Transl Res. 2015;7(8):1314-31.

[11] Allamand V, Campbell KP. Animal models for muscular dystrophy: Valuable tools for the development of therapies. Hum Mol Genet. 2000;9(16):2459-67.

[12] Lai Y, Duan D. Progress in gene therapy of dystrophic heart disease. Gene Ther. 2012;19(6):678-85.

[13] McGreevy JW, et al. Animal models of Duchenne muscular dystrophy: From basic mechanisms to gene therapy. Dis Model Mech. 2015;8(3):195-213.

[14] Willmann R, et al. Mammalian animal models for Duchenne muscular dystrophy. Neuromuscul Disord. 2009;19(4):241-9.

[15] Hor KN, et al. Prevalence and distribution of late gadolinium enhancement in a large population of patients with Duchenne muscular dystrophy: Effect of age and left ventricular systolic function. J Cardiovasc Magn Reson. 2013;15:107.

[16] Lu Q-L, et al. The status of exon skipping as a therapeutic approach to duchenne muscular dystrophy. Molecular Therapy. 2011;19(1):9-15.

[17] Fiszer A, Krzyzosiak WJ. RNA toxicity in polyglutamine disorders: Concepts, models, and progress of research. Journal of Molecular Medicine. 2013;91(6):683-91.

[18] Rajamohan D, et al. Current status of drug screening and disease modelling in human pluripotent stem cells. Bioessays. 2013;35(3):281-98.

[19] Nerbonne JM. Studying cardiac arrhythmias in the mouse - A reasonable model for probing mechanisms? Trends in Cardiovascular Medicine. 2004;14(3).

[20] Salama G, London B. Mouse models of long QT syndrome. Journal of Physiology-London. 2007;578(1).

[21] Denning C, et al. Cardiomyocytes from human pluripotent stem cells: From laboratory curiosity to industrial biomedical platform. Biochim Biophys Acta. 2015.

[22] Andruchov O, Andruchova O, Galler S. Fine-tuning of cross-bridge kinetics in cardiac muscle of rat and mouse by myosin light chain isoforms. Pflugers Arch. 2006;452(6):667-73.

[23] MacLennan DH, Kranias EG. Phospholamban: A crucial regulator of cardiac contractility. Nat Rev Mol Cell Biol. 2003;4(7):566-77.

[24] Modrek B, Lee CJ. Alternative splicing in the human, mouse and rat genomes is associated with an increased frequency of exon creation and/or loss. Nat Genet. 2003;34(2):177-80.

[25] Liao P, et al. Splicing for alternative structures of Cav 1.2 $\mathrm{Ca} 2+$ channels in cardiac and smooth muscles. Cardiovasc Res. 2005;68(2):197-203. 
[26] Weeland CJ, et al. Insights into alternative splicing of sarcomeric genes in the heart. J Mol Cell Cardiol. 2015;81:107-13.

[27] Yin Z, Ren J, Guo W. Sarcomeric protein isoform transitions in cardiac muscle: A journey to heart failure. Biochim Biophys Acta. 2015;1852(1):47-52.

[28] Price PS, Keenan RE, Swartout JC. Characterizing interspecies uncertainty using data from studies of antineoplastic agents in animals and humans. Toxicology and Applied Pharmacology. 2008;233(1):64-70.

[29] Wang L, et al. Stoichiometry of Gata4, Mef2c, and Tbx5 influences the efficiency and quality of induced cardiac myocyte reprogramming. Circulation Research. 2015;116(2):237-44.

[30] Fu J-D, et al. Direct reprogramming of human fibroblasts toward a cardiomyocyte-like state. Stem Cell Reports. 2013;1(3):235-47.

[31] Inagawa $\mathrm{K}$, et al. Induction of cardiomyocyte-like cells in infarct hearts by gene transfer of Gata4, Mef2c, and Tbx 5 . Circulation Research. 2012;111(9):1147-56.

[32] Zhang D, et al. Urine-derived stem cells: A novel and versatile progenitor source for cell-based therapy and regenerative medicine. Genes Dis. 2014;1(1):8-17.

[33] Zhou T, et al. Generation of human induced pluripotent stem cells from urine samples. Nat Protoc. 2012;7(12): 2080-9.

[34] Takahashi K, et al. Induction of pluripotent stem cells from adult human fibroblasts by defined factors. Cell. 2007;131(5):861-72.

[35] Warren L, et al. Highly efficient reprogramming to pluripotency and directed differentiation of human cells with synthetic modified mRNA. Cell Stem Cell. 2010;7(5): 618-30.

[36] Bladen CL, et al. The TREAT-NMD DMD Global database: Analysis of More Than 7000 Duchenne Muscular Dystrophy Mutations. Hum Mutat. 2015;36(4):395402.

[37] Soares FA, et al. International coordination of large-scale human induced pluripotent stem cell initiatives: Wellcome Trust and ISSCR workshops white paper. Stem Cell Reports. 2014;3(6):931-39.

[38] Waddington SN, et al. A broad overview and review of CRISPR-Cas technology and stem cells. Curr Stem Cell Rep. 2016;2:9-20.

[39] Braam SR, et al. Improved genetic manipulation of human embryonic stem cells. Nature Methods. 2008;5(5): 389-92.

[40] Kim YG, Cha J, Chandrasegaran S. Hybrid restriction enzymes: Zinc finger fusions to Fok I cleavage domain. Proc Natl Acad Sci U S A. 1996;93(3):1156-60.

[41] Porteus MH, Baltimore D. Chimeric nucleases stimulate gene targeting in human cells. Science. 2003;300(5620): 763.

[42] Cermak T, et al. Efficient design and assembly of custom TALEN and other TAL effector-based constructs for DNA targeting. Nucleic Acids Res. 2011;39(12):e82.

[43] Reyon D, et al. FLASH assembly of TALENs for high-throughput genome editing. Nat Biotechnol. 2012;30(5):460-5.

[44] Hsu PD, Lander ES, Zhang F. Development and applications of CRISPR-Cas9 for genome engineering. Cell. 2014;157(6):1262-78.

[45] Kim S, et al. Highly efficient RNA-guided genome editing in human cells via delivery of purified Cas9 ribonucleoproteins. Genome Res. 2014;24(6):1012-9.
[46] Sirenko O, et al. Assessment of beating parameters in human induced pluripotent stem cells enables quantitative in vitro screening for cardiotoxicity. Toxicol Appl Pharmacol. 2013;273(3):500-7.

[47] Pointon A, et al. Assessment of cardiomyocyte contraction in human-induced pluripotent stem cell-derived cardiomyocytes. Toxicol Sci. 2015;144(2):227-37.

[48] Braam SR, et al. Repolarization reserve determines drug responses in human pluripotent stem cell derived cardiomyocytes. Stem Cell Research. 2013;10(1):48-56.

[49] Harris K, et al. Comparison of Electrophysiological Data From Human-Induced Pluripotent Stem Cell-Derived Cardiomyocytes to Functional Preclinical Safety Assays. Toxicological Sciences. 2013;134(2):412-26.

[50] Nalos $\mathrm{L}$, et al. Comparison of the $\mathrm{I}(\mathrm{Kr})$ blockers moxifloxacin, dofetilide and E-4031 in five screening models of pro-arrhythmia reveals lack of specificity of isolated cardiomyocytes. British Journal of Pharmacology. 2012;165(2):467-78.

[51] Reynolds JG, et al. HER2-targeted liposomal doxorubicin displays enhanced anti-tumorigenic effects without associated cardiotoxicity. Toxicology and Applied Pharmacology. 2012;262(1):1-10.

[52] Sager PT, et al. Rechanneling the cardiac proarrhythmia safety paradigm: A meeting report from the Cardiac Safety Research Consortium. American Heart Journal. 2014;167(3):292-300.

[53] Lin B, et al. Modeling and study of the mechanism of dilated cardiomyopathy using induced pluripotent stem cells derived from individuals with Duchenne muscular dystrophy. Disease Models \& Mechanisms. 2015;8(5): 457-66.

[54] Sun N, et al. Patient-specific induced pluripotent stem cells as a model for familial dilated cardiomyopathy. Sci Transl Med. 2012;4(130):130ra47.

[55] Wu H, et al. Epigenetic Regulation of Phosphodiesterases $2 \mathrm{~A}$ and $3 \mathrm{~A}$ Underlies Compromised $\beta$-Adrenergic Signaling in an iPSC Model of Dilated Cardiomyopathy. Cell Stem Cell. 2015.

[56] Lan F, et al. Abnormal calcium handling properties underlie familial hypertrophic cardiomyopathy pathology in patient-specific induced pluripotent stem cells. Cell Stem Cell. 2013;12(1):101-13.

[57] Han L, et al. Study familial hypertrophic cardiomyopathy using patient-specific induced pluripotent stem cells. 2014;104:258-69.

[58] Carvajal-Vergara X, et al. Patient-specific induced pluripotent stem-cell-derived models of LEOPARD syndrome. Nature. 2010;465(7299):808-U12.

[59] Dudek J, et al. Cardiolipin deficiency affects respiratory chain function and organization in an induced pluripotent stem cell model of Barth syndrome. Stem Cell Research. 2013;11(2):806-19.

[60] Wang G, et al. Modeling the mitochondrial cardiomyopathy of Barth syndrome with induced pluripotent stem cell and heart-on-chip technologies. Nat Med. 2014;20(6):616-23.

[61] Ma D, et al. Generation of patient-specific induced pluripotent stem cell-derived cardiomyocytes as a cellular model of arrhythmogenic right ventricular cardiomyopathy. 2013;34:1122-33.

[62] Caspi O, et al. Modeling of Arrhythmogenic Right Ventricular Cardiomyopathy With Human Induced Pluripotent Stem Cells. Circulation: Cardiovascular Genetics. 2013;6(6):557-68. 
[63] Kim C, et al. Studying arrhythmogenic right ventricular dysplasia with patient-specific iPSCs. Nature. 2013;494(7435):105-10.

[64] Wen J-Y, et al. Maturation-Based Model of Arrhythmogenic Right Ventricular Dysplasia Using Patient-Specific Induced Pluripotent Stem Cells. Circulation Journal. 2015;79(7):1402-1408.

[65] Terrenoire C, et al. Induced pluripotent stem cells used to reveal drug actions in a long QT syndrome family with complex genetics. The Journal of General Physiology. 2013;141(1):61-72.

[66] Muntoni F. Cardiomyopathy in muscular dystrophies. Curr Opin Neurol. 2003;16(5):577-83.

[67] Kaspar RW, Allen HD, Montanaro F. Current understanding and management of dilated cardiomyopathy in Duchenne and Becker muscular dystrophy. J Am Acad Nurse Pract. 2009;21(5):241-9.

[68] Markham LW, et al. Abnormalities of diastolic function precede dilated cardiomyopathy associated with Duchenne muscular dystrophy. J Am Soc Echocardiogr. 2006;19(7):865-71.

[69] Verhaert D, et al. Cardiac involvement in patients with muscular dystrophies: Magnetic resonance imaging phenotype and genotypic considerations. Circ Cardiovasc Imaging. 2011;4(1):67-76.

[70] Yilmaz A, Sechtem U. Cardiac involvement in muscular dystrophy: Advances in diagnosis and therapy. Heart. 2012;98(5):420-29.

[71] Jung CB, et al. Dantrolene rescues arrhythmogenic RYR2 defect in a patient-specific stem cell model of catecholaminergic polymorphic ventricular tachycardia. Embo Molecular Medicine. 2012;4(3).

[72] Moretti A, et al. Patient-specific induced pluripotent stemcell models for long-QT syndrome. $\mathrm{N}$ Engl J Med. 2010;363(15):1397-409.

[73] Matsa E, et al. Allele-specific RNA interference rescues the long-QT syndrome phenotype in human-induced pluripotency stem cell cardiomyocytes. Eur Heart J. 2014;35(16):1078-87.

[74] Lahti AL, et al. Model for long QT syndrome type 2 using human iPS cells demonstrates arrhythmogenic characteristics in cell culture. Disease Models \& Mechanisms. 2012;5(2):220-30

[75] Matsa E, et al. Drug evaluation in cardiomyocytes derived from human induced pluripotent stem cells carrying a long QT syndrome type 2 mutation. Eur Heart J. 2011;32(8):952-62.

[76] Ma D, et al. Modeling type 3 long QT syndrome with cardiomyocytes derived from patient-specific induced pluripotent stem cells. Int J Cardiol. 2013;168(6): 5277-86.

[77] Davies KE, Nowak KJ. Molecular mechanisms of muscular dystrophies: Old and new players. Nature Reviews Molecular Cell Biology. 2006;7(10):762-73.

[78] Ervasti JM, Sonnemann KJ. Biology of the striated muscle dystrophin-glycoprotein complex. Int Rev Cytol. 2008;265:191-225.

[79] Endo T. Glycobiology of alpha-dystroglycan and muscular dystrophy. J Biochem. 2015;157(1):1-12.

[80] Hewitt JE. Abnormal glycosylation of dystroglycan in human genetic disease. Biochim Biophys Acta. 2009; 1792(9):853-61.

[81] Gilhuis HJ, et al. Nonmuscular involvement in merosinnegative congenital muscular dystrophy. Pediatr Neurol. 2002;26(1):30-6.
[82] Jones KJ, et al. The expanding phenotype of laminin alpha2 chain (merosin) abnormalities: Case series and review. J Med Genet. 2001;38(10):649-57.

[83] Yoshida-Moriguchi T, Campbell KP. Matriglycan: A novel polysaccharide that links dystroglycan to the basement membrane. Glycobiology. 2015;25(7):702-713.

[84] Van Erp C, et al. Timeline of cardiac dystrophy in 3-18month-old MDX mice. Muscle Nerve. 2010.

[85] Spurney CF, et al. Membrane sealant poloxamer p188 protects against isoproterenol induced cardiomyopathy in dystrophin deficient mice. BMC Cardiovasc Disord. 2011;11:20.

[86] Lorin C, Vogeli I, Niggli E. Dystrophic cardiomyopathy role of TRPV2 channels in stretch-induced cell damage. Cardiovasc Res. 2015.

[87] Koenig X, et al. Enhanced currents through L-type calcium channels in cardiomyocytes disturb the electrophysiology of the dystrophic heart. Am J Physiol Heart Circ Physiol. 2014;306(4):H564-73.

[88] Williams IA, Allen DG. Intracellular calcium handling in ventricular myocytes from mdx mice. Am J Physiol Heart Circ Physiol. 2007;292(2):H846-55.

[89] Townsend D, et al. Distinct pathophysiological mechanisms of cardiomyopathy in hearts lacking dystrophin or the sarcoglycan complex. Faseb J. 2011.

[90] Johnson EK, et al. Proteomic analysis reveals new cardiac-specific dystrophin-associated proteins. PLoS one. 2012;7(8):e43515.

[91] Montanaro F, et al. Laminin and alpha-dystroglycan mediate acetylcholine receptor aggregation via a MuSKindependent pathway. J Neurosci. 1998;18(4):1250-60.

[92] Montanaro F, Lindenbaum M, Carbonetto S. alphaDystroglycan is a laminin receptor involved in extracellular matrix assembly on myotubes and muscle cell viability. J Cell Biol. 1999;145(6):1325-40.

[93] Kobayashi YM, et al. Sarcolemma-localized nNOS is required to maintain activity after mild exercise. Nature. 2008;456(7221):511-5.

[94] Rando T. The dystrophin-glycoprotein complex, cellular signaling, and the regulation of cell survival in the muscular dystrophies. Muscle \& Nerve. 2001;24(12): 1575-94.

[95] Constantin B. Dystrophin complex functions as a scaffold for signalling proteins. Biochim Biophys Acta. 2014;1838(2):635-42.

[96] Miller G, et al. Preventing phosphorylation of dystroglycan ameliorates the dystrophic phenotype in mdx mouse. Hum Mol Genet. 2012;21(20):4508-20.

[97] Barton ER. Impact of sarcoglycan complex on mechanical signal transduction in murine skeletal muscle. Am J Physiol Cell Physiol. 2006;290(2):C411-9.

[98] Kumar A, et al. Loss of dystrophin causes aberrant mechanotransduction in skeletal muscle fibers. Faseb J. 2004;18(1):102-13.

[99] Jacobson C, et al. alpha-Dystroglycan functions in acetylcholine receptor aggregation but is not a coreceptor for agrin-MuSK signaling. J Neurosci. 1998;18(16): 6340-8.

[100] Dick E, et al. Exon skipping and gene transfer restore dystrophin expression in hiPSC-cardiomyocytes harbouring DMD mutations. Stem Cells Dev. 2013.

[101] Jung C, et al. Dystrophic cardiomyopathy: Amplification of cellular damage by $\mathrm{Ca} 2+$ signalling and reactive oxygen species-generating pathways. Cardiovascular Research. 2008;77(4):766-73. 
[102] Williams IA, Allen DG. Intracellular calcium handling in ventricular myocytes from mdx mice. American Journal of Physiology-Heart and Circulatory Physiology. 2007;292(2):H846-H855.

[103] Fraysse B, et al. Ca2+overload and mitochondrial permeability transition pore activation in living deltasarcoglycan-deficient cardiomyocytes. Am J Physiol Cell Physiol. 2010;299(3):C706-13.

[104] Yazawa M, et al. Using induced pluripotent stem cells to investigate cardiac phenotypes in Timothy syndrome. Nature. 2011;471(7337):230-4.

[105] Fatima A, et al. In vitro Modeling of Ryanodine Receptor 2 Dysfunction Using Human Induced Pluripotent Stem Cells. Cellular Physiology and Biochemistry. 2011;28(4).

[106] Itzhaki I, et al. Modeling of Catecholaminergic Polymorphic Ventricular Tachycardia With Patient-Specific Human-Induced Pluripotent Stem Cells. Journal of the American College of Cardiology. 2012;60(11):990-1000.

[107] Salzberg B, Davila H, Cohen L. Optical recording of impulses in individual neurones of an invertebrate central nervous system. Nature. 1973;246(5434):508-9.

[108] Liao M-LC, et al. Sensing Cardiac Electrical Activity With a Cardiac Myocyte-Targeted Optogenetic Voltage Indicator. Circulation Research. 2015;117(5):401-12.

[109] Allen D, et al. Stretch-Induced Membrane Damage in Muscle: Comparison of Wild-Type and mdx Mice. Muscle Biophysics: From Molecules To Cells. 2010;682:297-313.

[110] Deconinck N, Dan B. Pathophysiology of duchenne muscular dystrophy: Current hypotheses. Pediatric Neurology. 2007;36(1):1-7.

[111] Guan X, et al. Dystrophin-deficient cardiomyocytes derived from human urine: New biologic reagents for drug discovery. Stem Cell Res. 2014;12(2):467-80.

[112] Viola HM, et al. Impaired functional communication between the L-type calcium channel and mitochondria contributes to metabolic inhibition in the mdx heart. Proceedings of the National Academy of Sciences. 2014;111(28):E2905-E2914.

[113] Kikuchi C, et al. Comparison of cardiomycyte differentiation potential between type 1 diabetic donor- and nondiabetic donor-derived induced pluripotent stem cells. Cell Transplant. 2015;24(12):2491-2504.

[114] Bhattacharya SK, Johnson PL, Thakar JH. Reversal of impaired oxidative phosphorylation and calcium overloading in the in vitro cardiac mitochondria of CHF-146 dystrophic hamsters with hereditary muscular dystrophy. J Neurol Sci. 1993;120(2):180-6.

[115] Kuznetsov AV, et al. Impaired mitochondrial oxidative phosphorylation in skeletal muscle of the dystrophindeficient mdx mouse. Mol Cell Biochem. 1998;183(12):87-96.

[116] Yang C, et al. Concise Reviews: Cardiac Disease Modeling Using Induced Pluripotent Stem Cells. Stem Cells. 2015;33(9):2643-2651.

[117] Nakamori M, et al. Aberrantly spliced alpha-dystrobrevin alters alpha-syntrophin binding in myotonic dystrophy type 1. Neurology. 2008;70(9):677-85.

[118] Ueda K, et al. Syntrophin mutation associated with long QT syndrome through activation of the nNOS-SCN5A macromolecular complex. Proc Natl Acad Sci U S A. 2008;105(27):9355-60.

[119] Wu G, et al. alpha-1-syntrophin mutation and the long-QT syndrome: A disease of sodium channel disruption. Circ Arrhythm Electrophysiol. 2008;1(3):193-201.

[120] Pall GS, Johnson KJ, Smith GL. Abnormal contractile activity and calcium cycling in cardiac myocytes iso- lated from DMPK knockout mice. Physiol Genomics. 2003;13(2):139-46.

[121] Jung C, et al. Dystrophic cardiomyopathy: Amplification of cellular damage by $\mathrm{Ca} 2+$ signalling and reactive oxygen species-generating pathways. Cardiovasc Res. 2008;77(4):766-73.

[122] Macadangdang J, et al. Nanopatterned Human iPSC-based Model of a Dystrophin-Null Cardiomyopathic Phenotype. Cell Mol Bioeng. 2015;8(3):320-32.

[123] Ribeiro MC, et al. Functional maturation of human pluripotent stem cell derived cardiomyocytes in vitro-Correlation between contraction force and electrophysiology. Biomaterials. 2015;51:138-50.

[124] Liu J, et al. Atomic force mechanobiology of pluripotent stem cell-derived cardiomyocytes. PloS One. 2012; 7(5):e37559.

[125] Rodriguez ML, et al. Measuring the contractile forces of human induced pluripotent stem cell-derived cardiomyocytes with arrays of microposts. Journal of Biomechanical Engineering. 2014;136(5):051005.

[126] Kensah G, et al. Murine and human pluripotent stem cellderived cardiac bodies form contractile myocardial tissue in vitro. European Heart Journal. 2012:ehs349.

[127] Scott CW, et al. An impedance-based cellular assay using human iPSC-derived cardiomyocytes to quantify modulators of cardiac contractility. Toxicological Sciences. 2014;142(2):331-38.

[128] Eder A, et al. Human engineered heart tissue as a model system for drug testing. Advanced Drug Delivery Reviews. 2016;96:214-224.

[129] McNally EM, Sparano D. Mechanisms and management of the heart in myotonic dystrophy. Heart. 2011;97(13): 1094-100.

[130] Groh WJ, et al. Electrocardiographic abnormalities and sudden death in myotonic dystrophy type 1 . N Engl J Med. 2008;358(25):2688-97.

[131] Lasam G, et al. Symptomatic Trifascicular Block in Steinert's Disease: Is It Too Soon for a Pacemaker? Case Reports in Cardiology. 2016;2016:4.

[132] Brook JD, et al. Molecular basis of myotonic dystrophy: Expansion of a trinucleotide (CTG) repeat at the $3^{\prime}$ end of a transcript encoding a protein kinase family member. Cell. 1992;68(4):799-808.

[133] Meola G, Cardani R. Myotonic dystrophies: An update on clinical aspects, genetic, pathology, and molecular pathomechanisms. Biochimica et Biophysica Acta (BBA)Molecular Basis of Disease. 2015;1852(4):594-606.

[134] Kuyumcu-Martinez NM, Wang GS, Cooper TA. Increased steady-state levels of CUGBP1 in myotonic dystrophy 1 are due to PKC-mediated hyperphosphorylation. Mol Cell. 2007;28(1):68-78.

[135] Fardaei M, et al. In vivo co-localisation of MBNL protein with DMPK expanded-repeat transcripts. Nucleic Acids Res. 2001;29(13):2766-71.

[136] Kino Y, et al. MBNL and CELF proteins regulate alternative splicing of the skeletal muscle chloride channel CLCN1. Nucleic Acids Res. 2009;37(19):6477-90.

[137] Kimura T, et al. Altered mRNA splicing of the skeletal muscle ryanodine receptor and sarcoplasmic/endoplasmic reticulum $\mathrm{Ca} 2+-$ ATPase in myotonic dystrophy type 1 . Hum Mol Genet. 2005;14(15):2189-200.

[138] Philips AV, Timchenko LT, Cooper TA. Disruption of splicing regulated by a CUG-binding protein in myotonic dystrophy. Science. 1998;280(5364):737-41.

[139] Batra R, et al. Loss of MBNL leads to disruption of developmentally regulated alternative polyadenyla- 
tion in RNA-mediated disease. Mol Cell. 2014;56(2): 311-22.

[140] Wang ET, et al. Transcriptome-wide regulation of premRNA splicing and mRNA localization by muscleblind proteins. Cell. 2012;150(4):710-24.

[141] Rau F, et al. Misregulation of miR-1 processing is associated with heart defects in myotonic dystrophy. Nat Struct Mol Biol. 2011;18(7):840-5.

[142] Ravel-Chapuis A, et al. The RNA-binding protein Staufen1 is increased in DM1 skeletal muscle and promotes alternative pre-mRNA splicing. J Cell Biol. 2012;196(6):699-712.

[143] Pettersson OJ, et al. DDX6 regulates sequestered nuclear CUG-expanded DMPK-mRNA in dystrophia myotonica type 1. Nucleic Acids Res. 2014;42(11):7186-200.

[144] Tsoi H, et al. CAG expansion induces nucleolar stress in polyglutamine diseases. Proc Natl Acad Sci U S A. 2012;109(33):13428-33.

[145] $\mathrm{Zu} \mathrm{T}$, et al. Non-ATG-initiated translation directed by microsatellite expansions. Proc Natl Acad Sci U S A. 2011;108(1):260-5.

[146] Michel L, Huguet-Lachon A, Gourdon G. Sense and Antisense DMPK RNA Foci Accumulate in DM1 Tissues during Development. PLoS One. 2015;10(9):e0137620.

[147] Cho DH, et al. Antisense transcription and heterochromatin at the DM1 CTG repeats are constrained by CTCF. Mol Cell. 2005;20(3):483-9.

[148] Savić Pavićević D, et al. Molecular genetics and genetic testing in myotonic dystrophy type 1 . BioMed Research International. 2013;2013:13.

[149] Castel AL, Cleary JD, Pearson CE. Repeat instability as the basis for human diseases and as a potential target for therapy. Nature Reviews Molecular Cell Biology. 2010;11(3):165-170.

[150] McMurray CT. Mechanisms of trinucleotide repeat instability during human development. Nature Reviews Genetics. 2010;11(11):786-99.

[151] Du J, et al. Length-dependent CTG.CAG triplet-repeat expansion in myotonic dystrophy patient-derived induced pluripotent stem cells. Hum Mol Genet. 2013;22(25): 5276-87.

[152] Seriola A, et al. Huntington's and myotonic dystrophy hESCs: Down-regulated trinucleotide repeat instability and mismatch repair machinery expression upon differentiation. Hum Mol Genet. 2011;20(1):176-85.

[153] De Temmerman N, et al. CTG repeat instability in a human embryonic stem cell line carrying the myotonic dystrophy type 1 mutation. Mol Hum Reprod. 2008;14(7): 405-12.

[154] Dean NL, Tan SL, Ao A. Instability in the transmission of the myotonic dystrophy CTG repeat in human oocytes and preimplantation embryos. Fertil Steril. 2006;86(1): 98-105.

[155] De Temmerman N, et al. Intergenerational instability of the expanded CTG repeat in the DMPK gene: Studies in human gametes and preimplantation embryos. Am J Hum Genet. 2004;75(2):325-9.

[156] Wöhrle D, et al. Heterogeneity of DM kinase repeat expansion in different fetal tissues and further expansion during cell proliferation in vitro: Evidence for a causal involvement of methyl-directed DNA mismatch repair in triplet repeat stability. Human Molecular Genetics. 1995;4(7):1147-53.

[157] Martorell L, et al. Somatic instability of the myotonic dystrophy (CTG)n repeat during human fetal development. Hum Mol Genet. 1997;6(6):877-80.
[158] Yanovsky-Dagan S, et al. Uncovering the Role of Hypermethylation by CTG Expansion in Myotonic Dystrophy Type 1 Using Mutant Human Embryonic Stem Cells. Stem Cell Reports. 2015;5(2):221-31.

[159] Koshelev M, et al. Heart-specific overexpression of CUGBP1 reproduces functional and molecular abnormalities of myotonic dystrophy type 1 . Hum Mol Genet. 2010;19(6):1066-75.

[160] Chakraborty M, et al. Pentamidine rescues contractility and rhythmicity in a Drosophila model of myotonic dystrophy heart dysfunction. Dis Model Mech. 2015;8(12):1569-78.

[161] Xia G, et al. Generation of neural cells from DM1 induced pluripotent stem cells as cellular model for the study of central nervous system neuropathogenesis. Cell Reprogram. 2013;15(2):166-77.

[162] Mateizel I, et al. Derivation of human embryonic stem cell lines from embryos obtained after IVF and after PGD for monogenic disorders. Hum Reprod. 2006;21(2):503-11.

[163] Denis JA, et al. mTOR-dependent proliferation defect in human ES-derived neural stem cells affected by myotonic dystrophy type 1. J Cell Sci. 2013;126(Pt 8):1763-72.

[164] Xia G, et al. Genome modification leads to phenotype reversal in human myotonic dystrophy type 1 induced pluripotent stem cell-derived neural stem cells. Stem Cells. 2015;33(6):1829-38.

[165] Ketley A, et al. High-content screening identifies small molecules that remove nuclear foci, affect MBNL distribution and CELF1 protein levels via a PKC-independent pathway in myotonic dystrophy cell lines. Human Molecular Genetics. 2014;23(6):1551-62.

[166] Force T, Kolaja KL. Cardiotoxicity of kinase inhibitors: The prediction and translation of preclinical models to clinical outcomes. Nature Reviews Drug Discovery. 2011;10(2):111-26.

[167] Mioulane M, et al. Development of high content imaging methods for cell death detection in human pluripotent stem cell-derived cardiomyocytes. J Cardiovasc Transl Res. 2012;5(5):593-604.

[168] Sirenko O, et al. Multiparameter in vitro assessment of compound effects on cardiomyocyte physiology using iPSC cells. Journal of Biomolecular Screening. 2012:1087057112457590.

[169] Benders AA, et al. Myotonic dystrophy protein kinase is involved in the modulation of the $\mathrm{Ca} 2+$ homeostasis in skeletal muscle cells. J Clin Invest. 1997;100(6): 1440-7.

[170] Jacobs AE, et al. The calcium homeostasis and the membrane potential of cultured muscle cells from patients with myotonic dystrophy. Biochim Biophys Acta. 1990;1096(1):14-9.

[171] Botta A, et al. Altered Ca2+ homeostasis and endoplasmic reticulum stress in myotonic dystrophy type 1 muscle cells. Genes (Basel). 2013;4(2):275-92.

[172] Tang ZZ, et al. Muscle weakness in myotonic dystrophy associated with misregulated splicing and altered gating of $\mathrm{Ca}(\mathrm{V}) 1.1$ calcium channel. Hum Mol Genet. 2012;21(6):1312-24.

[173] Vihola A, et al. Altered expression and splicing of $\mathrm{Ca}(2+)$ metabolism genes in myotonic dystrophies DM1 and DM2. Neuropathol Appl Neurobiol. 2013;39(4):390-405.

[174] Oude Ophuis RJ, et al. A tail-anchored myotonic dystrophy protein kinase isoform induces perinuclear clustering of mitochondria, autophagy, and apoptosis. PLoS One. 2009;4(11):e8024. 
[175] O'Cochlain DF, et al. Transgenic overexpression of human DMPK accumulates into hypertrophic cardiomyopathy, myotonic myopathy and hypotension traits of myotonic dystrophy. Hum Mol Genet. 2004;13(20):2505-18.

[176] Gao Z, Cooper TA. Reexpression of pyruvate kinase M2 in type 1 myofibers correlates with altered glucose metabolism in myotonic dystrophy. Proc Natl Acad Sci U S A. 2013;110(33):13570-5.

[177] Wahbi K, et al. Electrophysiological study with prophylactic pacing and survival in adults with myotonic dystrophy and conduction system disease. Jama. 2012;307(12): 1292-301.

[178] Berul CI, et al. DMPK dosage alterations result in atrioventricular conduction abnormalities in a mouse myotonic dystrophy model. Journal of Clinical Investigation. 1999;103(4):R1.

[179] Echtelds MD, Bernsé A, Wieringa HHSB. Abnormal myotonic dystrophy protein kinase levels produce only mild myopathy in mice. Nature Genetics. 1996;13.

[180] Kaliman P, et al. Myotonic dystrophy protein kinase phosphorylates phospholamban and regulates calcium uptake in cardiomyocyte sarcoplasmic reticulum. Journal of Biological Chemistry. 2005;280(9):8016-21.

[181] Wahbi K, et al. Brugada syndrome and abnormal splicing of SCN5A in myotonic dystrophy type 1 . Arch Cardiovasc Dis. 2013;106(12):635-43.

[182] Pambrun T, et al. Myotonic dystrophy type 1 mimics and exacerbates Brugada phenotype induced by Nav1.5 sodium channel loss-of-function mutation. Heart Rhythm. 2014;11(8):1393-400.

[183] Algalarrondo V, et al. Abnormal sodium current properties contribute to cardiac electrical and contractile dysfunction in a mouse model of myotonic dystrophy type 1 . Neuromuscul Disord. 2015;25(4):308-20.

[184] Mounsey JP, et al. Phospholemman is a substrate for myotonic dystrophy protein kinase. J Biol Chem. 2000;275(30):23362-7.

[185] Kaprielian RR, et al. Distinct patterns of dystrophin organization in myocyte sarcolemma and transverse tubules of normal and diseased human myocardium. Circulation. 2000;101(22):2586-94.

[186] Bedada FB, Wheelwright M, Metzger JM. Maturation status of sarcomere structure and function in human iPSC-derived cardiac myocytes. Biochim Biophys Acta. 2015.

[187] Zhu R, et al. Physical developmental cues for the maturation of human pluripotent stem cell-derived cardiomyocytes. Stem Cell Res Ther. 2014;5(5):117.

[188] Birket MJ, et al. Expansion and patterning of cardiovascular progenitors derived from human pluripotent stem cells. Nature Biotechnology. 2015;33(9):970-79.

[189] Birket MJ, Mummery CL. Pluripotent stem cell derived cardiovascular progenitors-A developmental perspective. Developmental Biology. 2015;400(2):169-79.

[190] Devalla HD, et al. Atrial-like cardiomyocytes from human pluripotent stem cells are a robust preclinical model for assessing atrial-selective pharmacology. EMBO Molecular Medicine. 2015:e201404757.

[191] Kim C, et al. Non-cardiomyocytes influence the electrophysiological maturation of human embryonic stem cell-derived cardiomyocytes during differentiation. Stem Cells Dev. 2010;19(6):783-95.

[192] Tulloch NL, et al. Growth of engineered human myocardium with mechanical loading and vascular coculture. Circulation Research. 2011;109(1):47-59.
[193] Merkle FT, et al. Efficient CRISPR-Cas9-Mediated Generation of Knockin Human Pluripotent Stem Cells Lacking Undesired Mutations at the Targeted Locus. Cell Reports. 2015;11(6):875-83.

[194] Zhu Z, et al. A CRISPR/Cas-Mediated Selection-free Knockin Strategy in Human Embryonic Stem Cells. Stem Cell Reports. 2015;4(6):1103-1111.

[195] Chen Y, et al. Engineering human stem cell lines with inducible gene knockout using CRISPR/Cas9. Cell Stem Cell. 2015;17(2):233-44.

[196] Li HL, et al. Precise Correction of the Dystrophin Gene in Duchenne Muscular Dystrophy Patient Induced Pluripotent Stem Cells by TALEN and CRISPR-Cas9. Stem Cell Reports. 2015;4(1):143-54.

[197] Xie F, et al. Seamless gene correction of $\beta$-thalassemia mutations in patient-specific iPSCs using CRISPR/Cas9 and piggyBac. Genome Res. 2014;24(9):1526-33.

[198] Ousterout DG, et al. Correction of dystrophin expression in cells from duchenne muscular dystrophy patients through genomic excision of exon 51 by zinc finger nucleases. Mol Ther. 2015;23(3):523-32.

[199] Ousterout DG, et al. Reading frame correction by targeted genome editing restores dystrophin expression in cells from Duchenne muscular dystrophy patients. Molecular Therapy. 2013;21(9):1718-26.

[200] Lanphier E, et al. Don't edit the human germ line. Nature. 2015;519(7544):410-1.

[201] Liang P, et al. CRISPR/Cas9-mediated gene editing in human tripronuclear zygotes. Protein Cell. 2015;6(5):36372.

[202] Muntoni F, et al. New Perspectives on the Diagnosis and Management of Duchenne Muscular Dystrophy. European Neurological Review. 2015;10(1):73-8.

[203] Finsterer J, Stöllberger C. Cardiac involvement in Becker muscular dystrophy. Canadian Journal of Cardiology. 2008;24(10):786-92.

[204] Kirschner J, Lochmüller H. Sarcoglycanopathies. Handb Clin Neurol. 2011;101:41-6.

[205] Sveen M-L, et al. Cardiac involvement in patients with limb-girdle muscular dystrophy type 2 and Becker muscular dystrophy. Archives of Neurology. 2008;65(9): 1196-201.

[206] Poppe M, et al. Cardiac and respiratory failure in limbgirdle muscular dystrophy 2I. Annals of Neurology. 2004; 56(5):738-41.

[207] Quijano-Roy S, Sparks S, Rutkowski A. LAMA2Related Muscular Dystrophy, ed. R. Pagon, et al. 2012: GeneReviews ${ }^{\circledR}$ [Internet]. Seattle (WA): University of Washington, Seattle.

[208] Thornton CA. Myotonic dystrophy. Neurol Clin. 2014;32(3):705-19, viii.

[209] Scatenaa M. Heart rate variability in facioscapulohumeral muscular dystrophy. Functional Neurology. 2010;25(4):211-16.

[210] Siu CW, et al. Modeling of lamin A/C mutation premature cardiac aging using patient-specific induced pluripotent stem cells. Aging (Albany NY). 2012;4(11):803-22.

[211] Russo V, Nigro G. ICD role in preventing sudden cardiac death in Emery-Dreifuss muscular dystrophy with preserved myocardial function: 2013 ESC Guidelines on Cardiac Pacing and Cardiac Resynchronization Therapy. Europace. 2015;17(2):337.

[212] Kazuki Y, et al. Complete genetic correction of ips cells from Duchenne muscular dystrophy. Mol Ther. 2010;18(2):386-93. 
[213] Zatti S, et al. Complete restoration of multiple dystrophin isoforms in genetically corrected Duchenne muscular dystrophy patient-derived cardiomyocytes. Molecular Therapy- Methods \& Clinical Development. 2014;1.

[214] Darabi R, et al. Human ES- and iPS-Derived Myogenic Progenitors Restore DYSTROPHIN and Improve Contractility upon Transplantation in Dystrophic Mice. Cell Stem Cell. 2012;10(5):610-19.

[215] Lin B, et al. Modeling and Studying Mechanism of Dilated Cardiomyopathy Using Induced Pluripotent Stem Cells Derived From Duchenne Muscular Dystrophy (DMD) Patients. Disease Models and Mechanisms. 2015;8(3).
[216] Shoji E, et al. Early pathogenesis of Duchenne muscular dystrophy modelled in patient-derived human induced pluripotent stem cells. Sci Rep. 2015;5:12831.

[217] Abujarour R, et al. Myogenic differentiation of muscular dystrophy-specific induced pluripotent stem cells for use in drug discovery. Stem Cells Transl Med. 2014;3(2): 149-60.

[218] Marteyn A, et al. Mutant human embryonic stem cells reveal neurite and synapse formation defects in type 1 myotonic dystrophy. Cell Stem Cell. 2011;8(4):434-44. 